\title{
Completion Time Minimization in Fog-RANs using D2D Communications and Rate-Aware Network Coding
}

\author{
Mohammed S. Al-Abiad, Student Member, IEEE, and Md. Jahangir Hossain, \\ Senior Member, IEEE
}

\begin{abstract}
The device-to-device communication-aided fog radio access network, referred to as $D 2 D$-aided F-RAN, takes advantage of caching at enhanced remote radio heads (eRRHs) and D2D proximity for improved system performance. For D2D-aided F-RAN, we develop a framework that exploits the cached contents at eRRHs, their transmission rates/powers, and previously received contents by different users to deliver the requesting contents to users with a minimum completion time. Given the intractability of the completion time minimization problem, we formulate it at each transmission by approximating the completion time and decoupling it into two subproblems. In the first subproblem, we minimize the possible completion time in eRRH downlink transmissions, while in the second subproblem, we maximize the number of users to be scheduled on D2D links. We design two theoretical graphs, namely interference-aware instantly decodable network coding (IA-IDNC) and D2D conflict graphs to reformulate two subproblems as maximum weight clique and maximum independent set problems, respectively. Using these graphs, we heuristically develop joint and coordinated scheduling approaches. Through extensive simulation results, we demonstrate the effectiveness of the proposed schemes against existing baseline schemes. Simulation results show that the proposed two approaches achieve a considerable performance gain in terms of the completion time minimization.
\end{abstract}

\section{Index Terms}

Device-to-device communications, fog radio access networks, coordinated scheduling, network coding, power allocation, time-critical applications.

\section{INTRODUCTION}

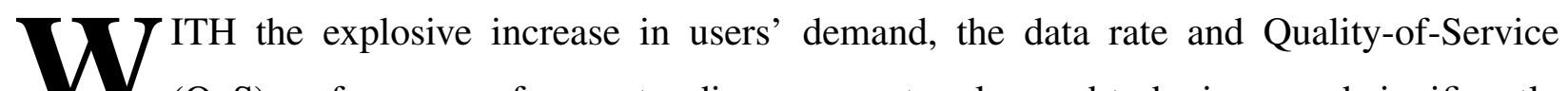
(QoS) performance of current radio access networks need to be improved significantly

Mohammed S. Al-Abiad and Md. Jahangir Hossain are with the School of Engineering, University of British Columbia, Kelowna, BC V1V 1V7, Canada (e-mail: m.saif@alumni.ubc.ca, jahangir.hossain@ubc.ca). 
[1]. Cloud radio access network (C-RAN) is a promising solution to improve the QoS for its users and support the exponentially growing demands [2]. The cloud base-station (CBS) in CRAN connects with distributed remote radio heads (RRHs) via fornthual links for cooperative transmission [3]-[6]. Since the requested contents are not cached at the RRHs, the capacity and delay constrained of fronthaul links limit the performance of C-RANs to meet the growing demand in 5G cellular networks [7]. Therefore, Fog-RAN (F-RAN) has been introduced that exploits both edge caching and C-RAN for carrying out content delivery effectively [8]. In F-RAN, the so called enhanced RRHs (eRRHs) support high caching capability.

In order to further improve the performance of F-RANs, implementing device-to-device (D2D) communications [9] in F-RAN is shown to be a potential technology in 5G and beyond. This integrated system is referred as D2D-aided F-RAN [13]. D2D-aided F-RAN system draws a remarkable benefit for reducing both users' contents delivery time and burden on fronthaul links. Thanks to the edge caching at the eRRHs and users' cooperation via D2D communications, this paper is focused on content delivery problem in D2D-aided F-RAN system. The content delivery problem of interest is motivated by immediate delivery of common popular contents for real-time applications, i.e., live video streaming. In particular, we study the scheduling of content delivery problem from both the eRRHs and potential transmitting users in D2D-aided F-RAN system using network coding (NC) [11].

The problem of delivering contents, i.e., a frame of delay-sensitive files, to a set of users with minimum possible delay has been a topic of research for a quite some time. This problem is referred as completion time minimization problem. Based on layer functionalities, existing $\mathrm{NC}$ solutions for this problem can be classified into upper-layer NC [12]-[19] and rate aware NC [20]-[27] methods. As their names indicate, upper layer NC algorithms focused only on $\mathrm{NC}$ at the network layer to minimize the number of transmissions. Rate aware NC approaches incorporate both upper and physical layers to minimize the completion time (in second) required to deliver requested files to all requesting users. The latter is more practically relevant as it involves the dynamic nature of wireless channels in the completion time optimization.

\section{A. Related Works and Challenges}

Related Works in Physical Layer: Most relevant works on C-RANs focused on scheduling users to RRHs in order to maximize sum-rate, e.g., [5], [6], [29]-[31]. The study in [5] was 
extended in [6] to include power allocation optimization for the radio resource blocks. However, these studies are agnostic to the available side information at network layer, i.e., requested and previously received contents by different users. As a result, each eRRH sends uncoded file that serves a single user. The term "uncoded file" is referred to file without NC. It has been observed that users tend to have a common interest in requesting same contents, especially popular videos, within a small interval of time [19]. This happens frequently in a hotspot, e.g., a playground, a public transport, a conference hall, and so on. In aforementioned schemes, the contents are transmitted without NC, which degrade the system performance. Therefore, a subclass of NC, namely the Instantly Decodable NC (IDNC), can be exploited to efficiently select a combination of contents (binary XOR combination) that can benefit a subset of interested users.

Related Works in Network Layer: The completion time minimization problem in IDNC-based networks was considered in different network settings, e.g., point-to-multipoint (PMP) [12], [14], D2D networks [16], [17], D2D F-RANs [18]. In particular, the authors of [12], [14], [16] proposed schemes to deliver the requested files by users with a minimum possible number of transmissions. Recently, in [18], a centralized D2D F-RAN scheme was proposed for completion time reduction. However, the aforementioned works considered IDNC from the perspective of network-layer. The main drawback is that the transmission rate of each radio resource block is selected based on the user with the weakest channel quality. This results in prolonged file reception time and thus, consumes the time resources of network. Therefore, considering both network layer coding and physical layer factors, such as transmission rate, is crucial, which is known as rate-aware IDNC (RA-IDNC) [20].

Related Works in RA-IDNC: With RA-IDNC, the completion time minimization problem needs a careful optimization of selecting the IDNC file and transmission rate of each radio resource, see for example [21]-[23], [26], [27]. The authors of [26] used RA-IDNC in C-RANs for completion time reduction. However, the authors assumed that all RRHs maintain a fixed transmit power level. Moreover, for synchronization purposes, the same transmission rate (i.e., the lowest transmission rate of all RRHs) is selected. This may violate the QoS rate guarantee and lead to a longer time for file transmission. Importantly, the proposed solution did not exploit the high capabilities of D2D communications. Recently, a cross-layer IDNC scheme was proposed for cloud offloading in F-RAN [28]. Inspired by [28], our work addresses the completion time minimization problem in D2D-aided F-RAN system using RA-IDNC and D2D communications. 
Challenges: The completion time minimization problem in D2D-aided F-RAN involves many factors, such as power levels of eRRHs, their cached files, users' limited coverage zones, their requested and previously received files, and their heterogeneous physical-layer capacities. Since all these combinatorial factors need to be jointly considered, such problem is intractable. Indeed, considering only power levels factor for solving a fixed schedule (without NC) problem is nonconvex [6], [29]-[31], [33]. To the best of the authors' knowledge, this work is the first attempt to solve the completion time minimization problem in D2D-aided F-RAN system while considering all above factors. Besides the intractability of the aforementioned factors, a key challenge to the problem is the use of IDNC codes in D2D-aided F-RAN as the objective of both techniques can be contradicting. Therefore, a balance among the conflicting effects of IDNC codes, scheduled users, and transmission rates/powers of eRRHs and users in D2D-aided F-RAN system is crucial to minimize the total frame delivery time.

\section{B. Contributions}

In this work, we tackle the completion time minimization problem in downlink D2D-aided F-RAN settings. To this end, we introduce a novel optimization framework taking network coding, rate/power optimization, potential D2D communications, and users' limited coverage zones into account. In the proposed framework, network-coded transmissions from both eRRHs and potential users are developed to deliver all files to all requesting users in the least amount of time. The main contributions of our work are summarized as follows.

1) For a D2D-aided F-RAN, we develop a framework where eRRHs and users collaborate to minimize the completion time. In particular, given the intractability of solving the completion time minimization problem over all possible future $\mathrm{NC}$ decisions, we reformulate the problem at each transmission with the constraints on user scheduling, their limited coverage zones, transmission rates, maximum power allocations, and QoS rate guarantee. By analyzing the problem, we decompose it into two subproblems.

2) The first subproblem aims to obtain the possible completion time in eRRHs transmissions through minimizing the transmission time. To solve it, we design an Interference-Aware IDNC (IA-IDNC) graph that efficiently solves the user scheduling and power allocation problem jointly under the completion time constraints. Based on this, the transmission time achieved by eRRHs is revealed for solving the second subproblem. Then, we introduce 
a new D2D conflict graph to heuristically solve the second subproblem, i.e., maximizing the number of users that can be scheduled on D2D links. The aforementioned graph-based solutions of the corresponding subproblems will be referred to as Joint Approach.

3) Since the IA-IDNC graph in the joint approach grows fast with the NC combinations in large network size, we propose an alternative and efficient low-complexity coordinated scheduling approach that solves the completion time problem using graph theoretic method.

4) We compare our proposed schemes with existing coded and uncoded (without NC) schemes. Selected numerical results demonstrate that the proposed schemes can effectively improve completion time performance.

The rest of this paper is organized as follows. In Section II we present an overview of the D2Daided F-RAN system. In Section III, we describe the NC model and analyze the transmission time for simplifying the expression of the completion time. The completion time minimization problem at each transmission is formulated and decomposed in Section IV. We solve the problem jointly in Section $\mathrm{V}$ and propose a relative low complexity approach in Section VI. Numerical results are presented in Section VII. Finally, Section VIII concludes the paper.

\section{Notations}

Matrices are shown by bold characters, e.g., C. Calligraphic letters denote sets and their corresponding capital letters denote the cardinalities of these sets, e.g., $N=|\mathcal{N}|$. Further, $\mathcal{P}(\mathcal{N})$ shows the power set of set $\mathcal{N}$ and $\mathcal{A} \times \mathcal{B}$ shows the Cartesian product of the two sets $\mathcal{A}$ and $\mathcal{B}$.

\section{SySTEM MODEL}

\section{A. System Overview}

We consider a D2D-aided F-RAN system, shown in Fig. 1, that consists of one cloud base station (CBS), $K$ single antenna enhanced remote radio heads (eRRHs), and $N$ users. The sets of eRRHs and users are denoted by $\mathcal{K}=\left\{e_{1}, e_{2}, \cdots, e_{K}\right\}, \mathcal{N}=\left\{u_{1}, u_{2}, \cdots, u_{N}\right\}$, respectively. The CBS is responsible for making the NC decisions, power allocation, delivering the instructions to eRRHs and transmitting users for executions. It also communicates with eRRHs through fronthaul links. Since users are allowed to transmit at a certain amount of power, each device has limited coverage zone, denoted by $\mathcal{Z}_{u_{i}}$, which represents the service area of the $u_{i}$-th user to transmit data within a circle of radius R. Note that user and device are used interchangeably 


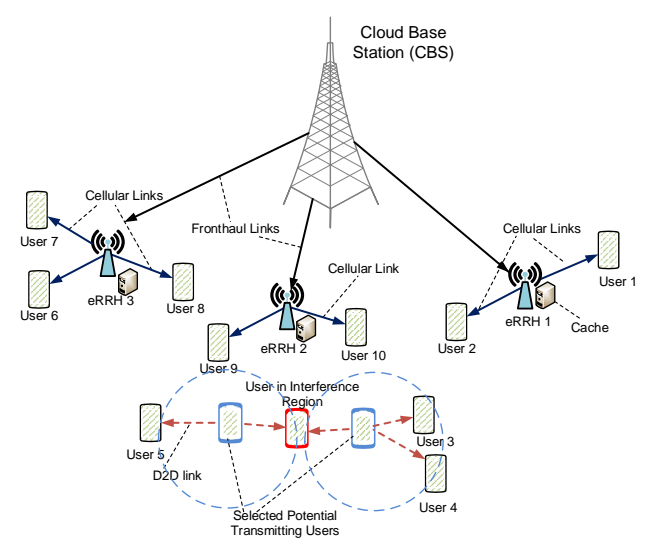

Fig. 1. Illustration of the D2D-aided F-RAN model with 13 users, 3 eRRHs and 1 CBS.

throughout this paper. The set of devices within the transmission range of the $u_{i}$-th device is defined by $\mathcal{Z}_{u_{i}}=\left\{u_{j} \in \mathcal{N} \mid d_{u_{i}, u_{j}}^{d 2 d} \leq \mathrm{R}\right\}$, where $d_{u_{i}, u_{j}}^{d 2 d}$ is the distance between the $u_{i}$-th and $u_{j}$-th devices. Devices can use the same frequency band and transmit encoded files simultaneously via D2D links. We assume there is a set of $F$ popular files, denoted by $\mathcal{F}=\left\{f_{1}, f_{2}, \cdots, f_{F}\right\}$. This data frame constitutes the set of most frequent requested files by the users within a given time duration in a hotspot area. Following the caching model in [28], the $e_{n}$-th eRRH caches a subset $\mathcal{C}_{e_{n}}$ that represents its cache, i.e., $\left|\mathcal{C}_{e_{n}}\right|=\mu F, \forall e_{n} \in \mathcal{K}$, where $0 \leq \mu \leq 1$ is the fractional cache size. Further, we assume that all eRRHs collectively cache all files in the frame, i.e., $\bigcup_{i=1}^{K} \mathcal{C}_{e_{n}}=\mathcal{F}$. The distribution of files among eRRHs is assumed to be given, and some common files can be cached in different eRRHs' caches.

In this paper, each device is assumed to be equipped with single antenna and used halfduplex channel. Thus, each device can access to either a D2D channel or cellular channel, and accordingly, it can either transmit or receive at a given time instant. Moreover, the allocated channels for D2D communications are assumed to be orthogonal (out-of-band) to those used by eRRHs, i.e., an overlay D2D communication model is adopted [13].

\section{B. Physical Layer Model}

The achievable rate at the $u_{i}$-th user when receives file from the $e_{n}$-th eRRH is given by $R_{e_{n}, u_{i}}^{c}=\log _{2}\left(1+\operatorname{SINR}_{e_{n}, u_{i}}(\mathbf{P})\right), \forall e_{n} \in \mathcal{K}, \forall u_{i} \in \mathcal{N}$, where $\operatorname{SINR}_{e_{n}, u_{i}}(\mathbf{P})$ is the corresponding signal-to-interference plus noise-ratio experienced by the $u_{i}$-th user when it is assigned to the 
$e_{n}$-th eRRH. This SINR is given by

$$
\operatorname{SINR}_{e_{n}, u_{i}}(\mathbf{P})=\frac{P_{e_{n}}\left|h_{e_{n}, u_{i}}^{c}\right|^{2}}{N_{0}+\sum_{e_{n^{\prime}} \in \mathcal{K}, e_{n^{\prime}} \neq e_{n}} P_{e_{n^{\prime}}}\left|h_{e_{n^{\prime}}, u_{i}}^{c}\right|^{2}},
$$

where $h_{e_{n}, u_{i}}^{c}$ denotes the channel gain between the $u_{i}$-th user and $e_{n}$-th eRRH, $N_{0}$ denotes the noise power, $P_{e_{n}}$ denotes the transmit power of $e_{n}$-th eRRH, and $\mathbf{P}=\left[P_{e_{n}}\right], \forall e_{n} \in \mathcal{K}$ is a row vector containing the power levels of the eRRHs in the considered network. The set of users' rates across all eRRHs can be written as $\mathcal{R}=\bigotimes_{\left(e_{n}, u_{i}\right) \in \mathcal{K} \times \mathcal{N}} R_{e_{n}, u_{i}}^{c}$, where the symbol $\bigotimes$ represents the product of the set of the achievable rates.

Similarly, let $h_{u_{k}, u_{i}}^{d 2 d}$ denote the channel gain for the D2D link between the $u_{k}$-th and $u_{i}$-th users and $Q_{u_{k}}$ denote the transmit power of the $u_{k}$-th user. Then, the achievable rate of D2D pair $\left(u_{k}, u_{i}\right)$ is given by $r_{u_{k}, u_{i}}^{d 2 d}=\log _{2}\left(1+\frac{Q_{u_{k}}\left|h_{u_{k}, u_{i}}^{d 2 d}\right|^{2}}{N_{0}+\sum_{u_{k^{\prime}} \in \mathcal{N}_{\text {tra }}, u_{k^{\prime}} \neq u_{k}} Q_{u_{k^{\prime}}}\left|h_{u_{k^{\prime}}, u_{i}}^{d 2 d}\right|^{2}}\right), \forall u_{k}, u_{k^{\prime}} \in$ $\mathcal{N}_{\text {tra }}$, and $u_{i} \in \mathcal{Z}_{u_{k}} \cap \mathcal{Z}_{u_{k^{\prime}}}$, where $\mathcal{N}_{\text {tra }}$ is the set of transmitting users via D2D links.

We assume $h_{e_{n}, u_{i}}^{c}$ and $h_{u_{k}, u_{i}}^{d 2 d}$ to be fixed during a single eRRH and D2D transmissions but change independently from one file transmission to another file transmission.

The channel capacities of all pairs of D2D links can be stored in an $N \times N$ capacity status matrix $(C S M) \mathbf{r}=\left[r_{u_{k}, u_{i}}\right], \quad \forall\left(u_{k}, u_{i}\right)$. Since $u_{k}$-th user does not transmit to itself and cannot transmit to other users outside its coverage zone, $r_{u_{k}, u_{k}}^{d 2 d}=0$ and $r_{u_{k}, u_{l}}^{d 2 d}=0, \forall u_{l} \notin \mathcal{Z}_{u_{k}}$.

\section{Network Coding And Completion Time Minimization}

\section{A. Network Coding in the Network-Layer}

We assume that users are interested in receiving the whole frame $\mathcal{F}$, and they have already acquired some files in $\mathcal{F}$ from prior broadcast transmissions (i.e., without NC) [19]. The previously acquired files by $u_{i}$-th user is denoted by the Has set $\mathcal{H}_{u_{i}}$, and its requested files is denoted by the Wants set, i.e., $\mathcal{W}_{u_{i}}=\mathcal{F} \backslash \mathcal{H}_{u_{i}}$. Taking advantage of the acquired and requested files by different users, each eRRH and D2D transmitter can perform XOR operation on these files and send the combined XORed files to the interested users. As such, the requested files are delivered to requesting users with minimum completion time. We use the subscript $t$ to represent the index of transmission/time slot, e.g., $t=1$ refers to the first transmission slot. After each transmission, each user feedbacks to the eRRHs and neighboring users an acknowledgment for each received file, and accordingly, the Has and Wants sets are updated by the CBS [18], [19]. The set of users having non-empty Wants sets at the $t$-th transmission slot is denoted by $\mathcal{N}_{w, t}$, 
which is defined as $\mathcal{N}_{w, t}=\left\{u_{i} \in \mathcal{N} \mid \mathcal{W}_{u_{i}, t} \neq \varnothing\right\}$. When a user receives its requested files, it can act as a D2D transmitter to provide its received files to the interested neighboring users.

Let $\mathrm{f}_{e_{n}, t}^{c}$ and $\mathrm{f}_{u_{k}, t}^{d 2 d}$ denote the XOR file combinations to be sent by the $e_{n}$-th eRRH and $u_{k}$-th D2D transmitter, respectively, to the sets of scheduled users $\mathrm{u}\left(\mathrm{f}_{e_{n}, t}^{c}\right)$ and $\mathrm{u}\left(\mathrm{f}_{u_{k}, t}^{d 2 d}\right)$ at the $t$-th transmission. For simplicity, the subscript transmission index $t$ is often omitted when it is clear from the context. These file combinations $\mathrm{f}_{e_{n}}^{c}$ and $\mathrm{f}_{u_{k}}^{d 2 d}$ are elements of the power sets $\mathcal{P}\left(\mathcal{C}_{e_{n}}\right)$ and $\mathcal{P}\left(\mathcal{H}_{u_{k}}\right)$, respectively. At every transmission slot $t$, each scheduled user in $\mathrm{u}\left(\mathrm{f}_{e_{n}}^{c}\right)$ can re-XOR $f_{e_{n}}^{c}$ with its previously received files to decode a new file. To ensure successful reception at the users, the maximum transmission rate of a particular transmitting $\mathrm{eRRH} / \mathrm{user}$ is equal to the minimum achievable capacity of its scheduled users. For discussion convenience, the term "targeted users" is referred to a set of scheduled users who receives an instantlydecodable transmission. Therefore, the set of targeted users by $e_{n}$-th eRRH is expressed as $\mathrm{u}\left(\mathrm{f}_{e_{n}}^{c}\right)=\left\{u_{i} \in \mathcal{N}_{w}|| \mathrm{f}_{e_{n}}^{c} \cap \mathcal{W}_{u_{i}} \mid=1\right.$ and $\left.R_{e_{n}}^{c} \leq R_{e_{n}, u_{i}}^{c}\right\}$. Similarly, for D2D transmissions, the set of targeted users by $u_{k}$-th D2D transmitter is expressed as $\mathrm{u}\left(\mathrm{f}_{u_{k}}^{d 2 d}\right)=\left\{u_{j} \in \mathcal{N}_{w}|| \mathrm{f}_{u_{k}}^{d 2 d} \cap \mathcal{W}_{u_{j}} \mid=\right.$ 1 and $u_{j} \in \mathcal{Z}_{u_{k}}$ and $\left.r_{u_{k}}^{d 2 d} \leq r_{u_{k}, u_{j}}^{d 2 d}\right\}$. Without loss of generality, the set of all targeted users when $\left|\mathcal{N}_{\text {tra }}\right| \mathrm{D} 2 \mathrm{D}$ transmitters transmit the set of combinations $\mathrm{f}^{d 2 d}\left(\mathcal{N}_{\text {tra }}\right)$ is represented by $\mathrm{u}\left(\mathrm{f}^{d 2 d}\left(\mathcal{N}_{\text {tra }}\right)\right)$, where $u_{k}, \mathrm{f}_{u_{k}}^{d 2 d}, \mathrm{u}\left(\mathrm{f}_{u_{k}}^{d 2 d}\right)$ are elements in $\mathcal{N}_{\text {tra }}, \mathrm{f}^{d 2 d}\left(\mathcal{N}_{\text {tra }}\right)$, and $\mathrm{u}\left(\mathrm{f}^{d 2 d}\left(\mathcal{N}_{\text {tra }}\right)\right)$, respectively.

\section{B. Transmission Time Analysis and Expression of the Completion Time}

This subsection provides an analysis of the transmission time for sending coded files from the eRRHs and D2D transmitters to a set of scheduled users, which leads to an expression of the completion time in D2D-aided F-RAN.

The transmission time for sending the coded file $f_{e_{n}}^{c}$ from the $e_{n}$-th eRRH with rate $R_{e_{n}}^{c}$ to the set of targeted users $\mathrm{u}\left(\mathrm{f}_{e_{n}}^{c}\right)$ is $T_{e_{n}}^{c}=\frac{B}{R_{e_{n}}}$ seconds, where $B$ is the size of the file in bits. Without loss of generality, let us assume that the $e_{n^{*}}$-th eRRH has the minimum rate at the $t$-th transmission slot that is denoted by $R_{e_{n^{*}}}$. The corresponding transmission duration is $T_{e_{n^{*}}}^{c}=\frac{B}{R_{e_{n^{*}}}}$ seconds. Since different eRRHs will have different transmission rates, they will have different transmission durations. Thus, the portion of the time that not being used by $e_{n^{\prime}}$ th eRRH at $t$-th transmission slot is referred to as the idle time of the $e_{n^{\prime}}$-th eRRH and denoted by $T_{e_{n^{\prime}} \text { idle. }}^{c}$. This idle time can be expressed as $T_{e_{n^{\prime}} \text { idle }}^{c}=\left(T_{e_{n^{*}}}^{c}-T_{e_{n^{\prime}}}^{c}\right)$ seconds. Such idle time can be exploited by the scheduled users of $e_{n^{\prime}}$ th $\mathrm{eRRH}$ via D2D links if it ensures the complete 
delivery of files, i.e., $T_{e_{n^{\prime}} \text { idle }}^{c} \geq T_{u_{m}}^{\mathrm{d} 2 \mathrm{~d}}$, where $T_{u_{m}}^{\mathrm{d} 2 \mathrm{~d}}=\frac{B}{r_{u_{k}}^{d 2 d}}$ is the transmission duration for sending $\mathrm{f}_{u_{m}}^{d 2 d}$ from the $u_{m}$-th D2D transmitter with adopted rate $r_{m}^{d 2 d}, \forall u_{m} \in \mathrm{u}\left(\mathrm{f}_{e_{n^{\prime}}}^{c}\right)$. The unscheduled users by the eRRHs can also use D2D links to transmit files, and accordingly, the transmission duration for sending $\mathrm{f}_{u_{k}}^{d 2 d}$ from the $u_{k}$-th D2D transmitter with adopted rate $r_{k}^{d 2 d}$ is $T_{u_{k}}^{d 2 d}=\frac{B}{r_{u_{k}}^{d 2 d}}$ seconds, $\forall u_{k} \notin \mathrm{u}\left(\mathrm{f}_{e_{n}}^{c}\right), \forall e_{n} \in \mathcal{K}$. Based on the above discussion, $u_{l}$-th user experiences one of three possible delays at each transmission, as shown in Fig. 2, and described below.

1) The time delay for $u_{l}$-th user receiving a non-instantly decodable transmission from $e_{n^{*}}$-th eRRH, this delay is $T_{e_{n^{*}}, u_{l}}^{c}, \forall u_{l} \notin \mathrm{u}\left(\mathrm{f}_{e_{n^{*}}}^{c}\right)$.

2) The time delay for $u_{l}$-th user receiving a non-instantly decodable transmission from $e_{n^{\prime}}$-th eRRH, this delay is $T_{e_{n^{\prime}}, u_{l}}^{c}, e_{n^{\prime}} \in \mathcal{K}$ and $u_{l} \notin \mathrm{u}\left(\mathrm{f}_{e_{n^{\prime}}}^{c}\right)$.

3) The time delay for $u_{l}$-th user being transmitting or receiving a non-instantly decodable transmission from any D2D transmitter in the set $\mathcal{N}_{\text {tra }}$, this delay is denoted as $T_{u_{k}, u_{l}}^{d 2 d}$, where $\left(u_{l}=u_{k}\right) \in \mathcal{N}_{\text {tra }}$ or $\left(u_{l} \notin \mathrm{u}\left(\mathrm{f}^{d 2 d}\left(\mathcal{N}_{\text {tra }}\right)\right)\right.$ and $\left.u_{k} \in \mathcal{N}_{\text {tra }}\right)$.

Note that for $u_{l^{-}}$-th user, $T_{e_{n^{\prime}}, u_{l}}^{c}$ is less than $T_{e_{n^{*}}, u_{l}}^{c}$ and $\max _{u_{k} \in \mathcal{N}_{\text {tra }}}\left(T_{u_{k}, u_{l}}^{d 2 d}\right)$. Thus, the maximum delay experienced by $u_{l}$-th user, which is not scheduled at the $t$-th transmission slot, is equal to $T_{\max , t}=\max \left(T_{e_{n^{*}}}^{c}, \max _{u_{k} \in \mathcal{N}_{\text {tra }}}\left(T_{u_{k}}^{d 2 d}\right)\right)$. Consequently, users that are not scheduled at transmission slot $t$, experience $T_{\max , t}$ seconds of delay in a cumulative manner defined as follows.

Definition 1. A user with non-empty Wants set experiences $T_{\max , t}$ seconds of time delay if it does not receive any requested file at $t$-th transmission slot. The accumulated time delay of $u_{l}$-th user is the sum of $T_{\max , t}$ seconds at each transmission until t-th transmission slot, and denoted by $\mathbb{D}_{u_{l}, t}$. It can be expressed as

$$
\mathbb{D}_{u_{l}, t}=\mathbb{D}_{u_{l}, t-1}+ \begin{cases}T_{\max , t} & \text { if } u_{l} \notin\left(\mathrm{u}\left(\mathrm{f}_{e_{n^{*}, t}^{c}}^{c} \cup \mathrm{u}\left(\mathrm{f}_{e_{n^{\prime}}, t}^{c}\right)\right), \forall e_{n^{*}}, e_{n^{\prime}} \in \mathcal{K}\right. \\ T_{\text {max }, t} & \text { if }\left(u_{l}=u_{k}\right) \in \mathcal{N}_{\text {tra }, t} \text { or }\left(u_{l} \notin \mathrm{u}\left(\mathrm{f}^{d 2 d}\left(\mathcal{N}_{\text {tra }, t}\right)\right) \text { and } u_{k} \in \mathcal{N}_{\text {tra }, t}\right)\end{cases}
$$

Definition 2. The completion time of $u_{l}$-th user, denoted by $\mathrm{T}_{u_{l}}$, is the total time required in seconds to receive all its requested files. The overall completion time $\mathrm{T}_{o}$ is the time required to receive all files by all users, and is given by $\mathrm{T}_{o}=\max _{u_{l} \in \mathcal{N}_{w}}\left\{\mathrm{~T}_{u_{l}}\right\}$.

Definition 3. A transmission schedule $\mathcal{S}=\left\{\left(\mathrm{f}_{e_{n}, t}^{c}, R_{e_{n}}^{c}\right),\left(u_{k}, \mathrm{f}_{u_{k}, t}^{d 2 d}, r_{u_{k}}^{d 2 d}\right)\right\}_{\forall t \in\{1,2, \ldots \ldots .,|\mathcal{S}|\}, \forall e_{n} \in \mathcal{K}, \forall u_{k} \in \mathcal{N}_{\text {tra }, t}}$ is a collection of transmitting eRRHs/D2D transmitters, their file combinations and adopted rates at every t-th transmission index to receive all files by all users. 
The completion time minimization problem in a D2D-aided F-RAN system can be expressed as follows

$$
\mathcal{S}^{*}=\arg \min _{\mathcal{S} \in \mathbf{S}}\left\{\mathrm{T}_{o}(\mathcal{S})\right\}=\arg \min _{\mathcal{S} \in \mathbf{S}}\left\{\max _{u_{l} \in \mathcal{N}_{w}}\left\{\mathrm{~T}_{u_{l}}(\mathcal{S})\right\}\right\},
$$

where $\mathcal{S}^{*}$ is the schedule that optimally minimizes the overall completion time and $\mathbf{S}$ is the set of all possible transmission schedules. The follwoing theorem expresses the optimal schedule $\mathcal{S}^{*}$ in terms of time delay defined in definition 1 .

Theorem 1. The optimal schedule $\mathcal{S}^{*}$ that minimizes the overall completion time in a D2D-aided F-RAN system can be written as follows

$$
\mathcal{S}^{*}=\arg \min _{\mathcal{S} \in \mathbf{S}}\left\{\max _{u_{l} \in \mathcal{N}_{w}}\left\{\frac{B \cdot\left|\mathcal{W}_{u_{l}, 0}\right|}{\tilde{R}_{u_{l}}(\mathcal{S})}+\mathbb{D}_{u_{l}}(\mathcal{S})\right\}\right\},
$$

where $\left|\mathcal{W}_{u_{l}, 0}\right|$ is the initial Wants size of $u_{l}$-th user, $\mathbb{D}_{u_{l}}(\mathcal{S})$ is the accumulative time delay of $u_{l}$-th user in schedule, and $\tilde{R}_{u_{l}}(\mathcal{S})$ is the harmonic mean of the transmission rates of transmissions that are instantly decodable for $u_{l}$-th user in schedule $\mathcal{S}$.

Proof. The proof of Theorem 1 is omitted in this paper due to the space limitation and only a sketch of the proof is given as follows. We first show that the completion time can be expressed as the sum of instantly and non-instantly decodable transmission times from $|\mathcal{K}|$ and $\left|\mathcal{N}_{\text {tra }}\right|$ transmitters via cellular and D2D links, respectively. Afterward, we need to proof that the number of instantly decodable transmissions to $u_{l}$-th user is equal to the number of its requested files $\left|\mathcal{W}_{u_{l}, 0}\right|$ and the number of non-instantly decodable transmissions matches the time delay in definition 1. Finally, we extend the results of the optimal schedule in Theorem 1 in [20] that used in PMP system with a single transmitter to the coordinated D2D-aided F-RAN setting with multiple transmitters.

Solving the completion time problem in (3) optimally is intractable [26]. In fact, the transmission schedule at the current transmission slot does not depend only on the future transmission schedules, but also on users' achievable capacities and eRRHs' transmit powers. Therefore, we pay our special attention to solve such problem at each transmission, where files are transmitted with high transmission rates. If some eRRHs cannot send XOR files to a set of users with the rate threshold $R_{\mathrm{th}}$, these users can be scheduled on D2D links. To this end, our main objective is to minimize the completion time at each transmission, known as the anticipated completion time [19], through minimizing the time delay. This anticipated user's completion time at each 


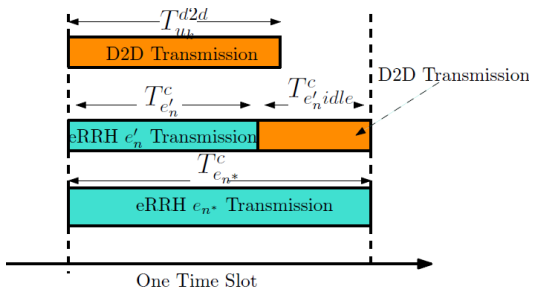

Fig. 2. Transmission time structure for eRRHs and potential D2D transmitters for one time slot.

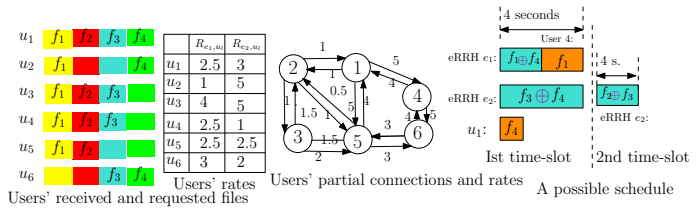

Fig. 3. D2D-aided F-RAN system containing 6 users and their corresponding requested/received files and rates.

transmission in D2D-aided F-RAN system is given in the next corollary.

Corollary 1. The anticipated completion time of $u_{l}$-th user at $t$-th transmission slot is given by

$$
\mathrm{T}_{u_{l}, t} \approx \frac{B \cdot\left|\mathcal{W}_{u_{l}, 0}\right|}{\tilde{R}_{u_{l}, t}}+\mathbb{D}_{u_{l}, t},
$$

where $\mathbb{D}_{u_{l}, t}$ is the accumulative transmission delay as given in $(2)$, and $\tilde{R}_{u_{l}, t}$ is the harmonic mean of the transmission rates that are instantly decodable for $u_{l}$-th user until $t$-th transmission.

The anticipated completion time in Corollary 1 depends on the number of requested files by $u_{l}$-th user, its accumulated time delay and harmonic mean $\tilde{R}_{u_{l}, t}$. Clearly, this metric is intimately related to the duration of time that all files are delivered to all users, which can be illustrated in the following example.

Example 1: This example considers the model in Fig. 3 that consists of 2 eRRHs, 6 users, users' received and requested files and their rates. For example, $u_{2}$ receives $f_{1}, f_{4}$ and requests $f_{2}, f_{3}$. The sets of files that stored in eRRHs' caches are $\mathcal{C}_{e_{1}}=\left\{f_{1}, f_{4}, f_{3}\right\}, \mathcal{C}_{e_{2}}=\left\{f_{2}, f_{3}, f_{4}\right\}$. Each file is assumed to have a size of 10 bits. To minimize the completion time for this example, one possible schedule is given as follows.

First time slot: The $e_{1}$-th and $e_{2}$-th eRRHs transmit $\mathrm{f}_{e_{1}, 1}^{c}=f_{1} \oplus f_{4}$ and $\mathrm{f}_{2}^{c}=f_{3} \oplus f_{4}$ with rates $R_{e_{1}}^{c}=2.5$ and $R_{e_{2}}^{c}=5$ bits/s, respectively, to the sets $\mathrm{u}\left(\mathrm{f}_{e_{1}, 1}^{c}\right)=\left\{u_{4}, u_{6}\right\}$ and $\mathrm{u}\left(\mathrm{f}_{e_{2}}^{c, 1}\right)=\left\{u_{2}, u_{3}\right\}$. The $u_{1}$-th user transmits $\mathrm{f}_{u_{1}, 1}^{d 2 d}=f_{4}$ with rate $r_{u_{1}}^{d 2 d}=5$ bits/s to the set $\mathrm{u}\left(\mathrm{f}_{u_{1}, 1}^{d 2 d}\right)=\left\{u_{5}\right\}$. Given this, we have the following transmission durations of $e_{1}$-th eRRH, $e_{2}$-th eRRH, and $u_{1}$-th transmitting user, respectively: $T_{e_{1}}^{c}=\frac{10}{2.5}=4, T_{e_{1}}^{c}=\frac{10}{5}=2, T_{u_{1}}^{d 2 d}=\frac{10}{5}=2$ seconds. Since user $u_{4}$ receives $f_{4}$ from $e_{1}$-th eRRH in 4 seconds, it can use the idle time of $e_{1}$-th eRRH, i.e., $T_{e_{1} \text { idle }}^{c}=2$ seconds, to send $f_{2}$ to $u_{6}$ with rate $r_{u_{4}}^{d 2 d}=5 \mathrm{bits} / \mathrm{s}$. Therefore, the updated Wants sets after the first time slot are: $\mathcal{W}_{u_{2}, 1}=\left\{f_{2}\right\}, \mathcal{W}_{u_{3}, 1}=\varnothing, \mathcal{W}_{u_{4}, 1}=\varnothing, \mathcal{W}_{u_{5}, 1}=\left\{f_{3}\right\}, \mathcal{W}_{u_{6}, 1}=\varnothing$. Note that $T_{\max , 1}=\max \left(T_{e_{1}}^{c}, T_{e_{2}}^{c}, T_{u_{1}}^{d 2 d}\right)=4$ seconds. 
Second time slot: The $e_{2}$-th eRRH transmits $f_{e_{2}}^{c, 2}=f_{2} \oplus f_{3}$ with rate $R_{e_{2}}^{c}=2.5$ bits/s to the set $\mathrm{u}\left(\mathrm{f}_{e_{2}}^{c, 2}\right)=\left\{u_{2}, u_{5}\right\}$ which requires transmission time $T_{e_{2}}^{c}=T_{\max , 2}=\frac{10}{2.5}=4$ seconds. By the end of second time slot, all users will have their wanted files. Therefore, the total transmission time is $T_{\max , 1}+T_{\max , 2}=8$ seconds.

\section{Problem Formulation And Problem Decomposition}

\section{A. Problem Formulation}

In order to minimize the completion time at each transmission slot, we need to develop a rate-aware network coding framework that decides: i) the adopted transmission rate/power at the $e_{n}$-th eRRH, $\left\{R_{e_{n}}^{c}, P_{e_{n}}\right\}$, to transmit its XOR combination $\mathrm{f}_{e_{n}, t}^{c}$ to a set of targeted users u $\left(\mathrm{f}_{e_{n}, t}^{c}\right)$, $\forall e_{n} \in \mathcal{K}$, and ii) the set of D2D transmitters $\mathcal{N}_{\text {tra }, t}$ for sending $\mathrm{f}_{u_{k}, t}^{d 2 d}$ to the users $\mathrm{u}\left(\mathrm{f}_{u_{k}}^{d 2 d, t}\right)$, and their adopted transmission rates $r_{u_{k}}^{d 2 d}, \forall u_{k} \in \mathcal{N}_{\text {tra, }, t}$. As such, all files are delivered to all users with minimum completion time. Therefore, the completion time minimization problem in D2D-aided F-RAN system can be formulated as

P1 : $\min _{\mathbf{f}_{e_{n}, t}^{c}, \mathbf{f}_{u_{k}, t}^{d 2 d}, P_{e_{n}}, \mathcal{N}_{\text {tra }, t} \in \mathcal{P}(\mathcal{N})}\left\{\max _{\mathrm{u}_{1} \in \mathcal{N}_{\mathrm{w}, \mathrm{t}}} \mathrm{T}_{\mathrm{u}_{1}, \mathrm{t}}\right\}$

subject to $\left\{\begin{array}{l}\mathrm{C} 1: \mathrm{u}\left(\mathrm{f}_{e_{n}, t}^{c}\right) \cap \mathrm{u}\left(\mathrm{f}_{e_{n^{\prime}}, t}^{c}\right)=\varnothing, \forall e_{n} \neq e_{n^{\prime}} \in \mathcal{K} ; \\ \mathrm{C} 2: \mathrm{u}\left(\mathrm{f}_{u_{k}, t}^{d 2 d}\right) \cap \mathrm{u}\left(\mathrm{f}_{u_{k^{\prime}}, t}^{d 2 d}\right)=\varnothing \& \mathrm{u}\left(\mathrm{f}_{u_{k}, t}^{d 2 d}\right) \cap \mathrm{u}\left(\mathrm{f}_{e_{n}, t}^{c}\right)=\varnothing, \forall u_{k} \neq u_{k^{\prime}} \in \mathcal{N}_{\text {tra }, t}, e_{n} \in \mathcal{K} ; \\ \mathrm{C} 3: r_{u_{k}}^{d 2 d} \cdot T_{e_{n^{\prime}}}^{c} \text { ddle } \geq B, \forall u_{k} \in \mathcal{N}_{\text {tra }, t}, \forall e_{n^{\prime}} \in \mathcal{K} ; \\ \mathrm{C} 4: \mathrm{f}_{e_{n}, t}^{c} \subseteq \mathcal{P}\left(\mathcal{C}_{e_{n}}\right) \& \mathrm{f}_{u_{k}, t}^{d 2 d} \subseteq \mathcal{P}\left(\mathcal{H}_{u_{k}, t}\right), \forall\left(e_{n}, u_{k}\right) \in \mathcal{K} \times \mathcal{N}_{\text {tra }, t} ; \\ \mathrm{C} 5: 0 \leq P_{e_{n}} \leq P_{\text {max }}, \forall e_{n} \in \mathcal{K} ; \mathrm{C} 6: R_{e_{n}}^{c} \geq R_{\text {th }} ; \mathrm{C} 7: r_{u_{k}}^{d 2 d} \geq R_{\text {th }}, \forall e_{n} \in \mathcal{K}, \forall u_{k} \in \mathcal{N}_{\text {tra }, t} .\end{array}\right.$ The constraints are explained as follows. $\mathrm{Cl}$ states that the set of scheduled users to all eRRHs are disjoint, i.e., each user must be scheduled to only one eRRH. C2 makes sure that each user can be scheduled to only one potential D2D transmitter and no user can be scheduled to a D2D transmitter and eRRH at the same time instant. C3 ensures the successful delivery of files from D2D transmissions within the idle time of the eRRHs. C4 ensures that all files to be combined using XOR operation at all eRRHs and D2D transmitters are stored in their Caches and Has sets, respectively. C5 bounds the maximum transmit power of each eRRH, and C6 and C7 satisfy the minimum transmission rates required to meet the QoS rate requirement $R_{\mathrm{th}}$.

The optimization problem in $\mathrm{P} 1$ contains the NC scheduling parameters $\mathrm{u}\left(\mathrm{f}_{e_{n}, t}^{c}\right), \mathrm{u}\left(\mathrm{f}_{u_{k}, t}^{d 2 d}\right), \forall e_{n} \in$ $\mathcal{K}, \forall u_{k} \in \mathcal{N}_{\text {tra, },}$, power allocations of eRRHs $P_{e_{n}}, \forall e_{n} \in \mathcal{K}$, potential set of transmitting users 
$\mathcal{N}_{\text {tra }, t}$ and their transmission rates. We can readily show that problem P1 is NP-hard and intractable [32]. However, by analyzing the problem, we can decompose it into two subproblems and solve them individually and efficiently using graph theory technique [35].

\section{B. Problem Decomposition}

Since the main objective is to minimize the maximum completion time of users, which depends on the time delay increase at each transmission slot, we can first focus on minimizing the transmission duration for the eRRH-user NC transmissions. In particular, we can get the possible completion time by jointly optimizing the NC user scheduling and power allocations of eRRHs. The mathematical formulation for minimizing the transmission duration for eRRH-user $\mathrm{NC}$ transmissions can be expressed as

$$
\begin{aligned}
& \text { P2 : } \min _{0 \leq P_{e_{n}} \leq P_{\max }} \mathrm{T}_{\mathrm{e}_{\mathrm{n}}, \mathrm{t}}^{\mathrm{c}} \\
& \text { subject to }\left\{\begin{array}{l}
\mathrm{u}\left(\mathrm{f}_{e_{n}, t}^{c}\right) \cap \mathrm{u}\left(\mathrm{f}_{e_{n^{\prime}}, t}^{c}\right)=\varnothing, \forall e_{n} \neq e_{n^{\prime}} \in \mathcal{K} ; \\
\mathrm{f}_{e_{n}, t}^{c} \subseteq \mathcal{P}\left(\mathcal{C}_{e_{n}}\right) ; R_{e_{n}}^{c} \geq R_{\mathrm{th}}, \forall e_{n} \in \mathcal{K} .
\end{array}\right.
\end{aligned}
$$

Note that this subproblem contains users' associations and power allocation variables and a joint solution will be developed in Section V-B.

After obtaining the possible transmission duration from eRRH-user NC transmissions, denoted by $T_{e_{n^{*}, t}}^{c}$ of $e_{n^{*}}$-th eRRH, by solving P2, we can now formulate the second subproblem. In particular, we can maximize the number of users $Z_{t}$ that are not been scheduled to the eRRHs $\mathcal{N}_{w, t} \backslash \mathrm{u}\left(\mathrm{f}_{e_{n^{*}, t}}^{c}\right)$ within $T_{e_{n^{*}, t}}^{c}$ by using D2D communication. In addition, users being scheduled to $e_{n^{\prime}}$-th eRRH from subproblem P2, have the opportunity to be scheduled on D2D links within the idle times of their corresponding eRRHs at the $t$-th transmission slot, $\forall e_{n^{\prime}} \neq e_{n^{*}} \in \mathcal{K}$. Therefore, the second subproblem of maximizing the number of users to be scheduled on D2D links can be expressed as follows

$$
\begin{aligned}
& \text { P3 : } \max _{\substack{\mathcal{N}_{\text {tra }, t} \in \mathcal{P}\left(\mathcal{N} \backslash \mathbf{u}\left(f_{e_{n^{*}, t}^{c}}^{c}\right)\right) \\
\mathbf{f}_{u_{k}, t}^{d 2 d} \subseteq \mathcal{P}\left(\mathcal{H}_{u_{k}, t}\right)}} \mathrm{Z}_{\mathrm{t}} \\
& \text { subject to }\left\{\begin{array}{l}
(\mathrm{C} 2) ; r_{u_{k}}^{d 2 d} \cdot\left(T_{e_{n^{*}}, t}^{c}-T_{e_{n^{\prime}}, t}^{c}\right) \geq B, \forall u_{k} \in \mathcal{N}_{\text {tra }, t}, \quad \forall\left(e_{n^{*}}, e_{n^{\prime}}\right) \in \mathcal{K} ; \\
T_{u_{k}}^{d 2 d} \leq T_{e_{n^{*}, t}}^{c}, \forall u_{k} \in \mathcal{N}_{\text {tra }, t} ; \\
\left|\mathrm{u}\left(\mathrm{f}^{d 2 d}\left(\mathcal{N}_{\text {tra }, t}\right)\right)\right|+\left|\mathcal{N}_{\text {tra }, t}\right| \leq Z_{t} .
\end{array}\right.
\end{aligned}
$$


The constraint $\mathrm{C} 3$ in $\mathrm{P} 1$ is rewritten as the second constraint in P3 since we know $T_{e_{n^{*}}, t}^{c}$. Fourth constraint states that the transmission duration of any D2D transmitter should be less than or equal to $T_{e_{n^{*}, t}}^{c}$. The last constraint is the maximum number limitation of scheduled users on D2D links. It can be easily observed that P3 is a D2D scheduling problem that considers selection of D2D transmitters, their NC files and transmission rates.

\section{Completion Time Minimization: Joint ApPROACH}

In this section, we propose a joint approach to solve the subproblems in P2 and P3 using designed interference-aware IDNC and new D2D conflict graphs, respectively. Specifically, we design interference-aware IDNC in the first subsection to solve the subproblem P2 in the second subsection. We then introduce a new D2D conflict graph to solve the subproblem P3 as shown in the third and fourth subsections, respectively.

\section{A. Subproblem P2 Transformation: Interference Aware-IDNC Graph}

Interference-Aware IDNC (IA-IDNC) graph, denoted by $\mathcal{G}_{\text {IA-IDNC }}(\mathcal{V}, \mathcal{E})$, is designed to systematically select an IDNC combination, transmission rate, and power allocation of each eRRH at the $t$-th transmission slot. Unlike the graph in [26] that resulted in one rate for fixed power eRRHs, our designed IA-IDNC graph leads to different transmission rates/powers from different eRRHs. This gives flexibility to each eRRH to choose its IDNC combination and transmission rate that satisfy a set of scheduled users.

Consider generating all possible associations (pairs) representing users and their corresponding requested files that cached by $e_{n}$-th eRRH, denoted by $\mathcal{A}_{e_{n}}=\mathcal{N}_{w} \times \mathcal{C}_{e_{n}}$, i.e., $a \in \mathcal{A}_{e_{n}}=\left(u_{l}, f_{h}\right)$ represents the association of $u_{l}$-th user and its $f_{h}$-th requested file. The corresponding files of a set of associations in $\mathcal{A}_{e_{n}}$ can be encoded into one IDNC combination if these files are instantly decodable to the corresponding associated users. The set of all IDNC combinations is denoted by $\mathcal{A}_{e_{n}, \text { IDNC }}$. In particular, the corresponding files of any two different associations $a \in \mathcal{A}_{e_{n}}$ and $a^{\prime} \in \mathcal{A}_{e_{n}}$ are encoded if one of the following IDNC conditions is satisfied.

- IDNC-C1: $u_{l, a} \neq u_{l^{\prime}, a^{\prime}}$ and $f_{h, a}=f_{h, a^{\prime}}$ This condition represents that the same file $f_{h}$ is requested by two distinct users $u_{l}$ and $u_{l^{\prime}}$.

- IDNC-C2: $u_{l, a} \neq u_{l^{\prime}, a^{\prime}}$ and $f_{h^{\prime}, a^{\prime}} \in \mathcal{H}_{u_{l}, a}$ and $f_{h, a} \in \mathcal{H}_{u_{l^{\prime}}, a^{\prime}}$. This condition represents that different files $f_{h^{\prime}}$ and $f_{h}$ are requested by two different users $u_{l^{\prime}}$ and $u_{l}$, respectively. 
Meanwhile, the requested file of each user is in the Has set of the user in the other association. We use $l, a$ in $u_{l, a}$ as subscripts to represent $u_{l}$-th user in $a$-th association.

For example, the element $\mathrm{a}=\left(\mathrm{f}_{e_{n}}^{c}, \mathrm{u}\left(\mathrm{f}_{e_{n}}^{c}\right)\right) \in \mathcal{A}_{e_{n}, \text { IDNC }}$ represents the set of scheduled users $\mathrm{u}\left(\mathrm{f}_{e_{n}}^{c}\right)$ that will receive the IDNC combination $\mathrm{f}_{e_{n}}^{c}$ from $e_{n}$-th eRRH.

Let $\mathcal{S}_{e_{n}}$ be the set of all possible associations between the IDNC combinations $\mathcal{A}_{e_{n}, \text { IDNC }}$ and achievable capacities $\mathcal{R}_{e_{n}} \subset \mathcal{R}$, i.e., $\mathcal{S}_{e_{n}}=\mathcal{A}_{e_{n}, \text { IDNC }} \times \mathcal{R}_{e_{n}}$. In other words, $\mathbf{S}=(\mathrm{a}, R) \in \mathcal{S}_{e_{n}}$ is a schedule that consists of a set of associations representing the IDNC combination, set of scheduled users, and rate $R$ of $e_{n}$-th eRRH, i.e., $\mathbf{S}=(\mathrm{a}, R)=s_{1}, s_{2}, \cdots, s_{\mid \mathbf{S}} \mid$, where $|\mathbf{S}|$ is the total number of scheduled users in $\mathbf{S}$. Note that $s_{1}$ represents one user, one file, and rate of $e_{n}$-th eRRH. Now, any two associations $s_{1} \in \mathbf{S}, s_{2} \in \mathbf{S}$ representing the $e_{n}$-th eRRH should have an equal adopted rate that is greater than or equal to $R_{\mathrm{th}}$. That is, the Rate Condition (RC) is satisfied $R_{s_{1}}=R_{s_{2}}$ and $R_{s_{1}} \geq R_{\mathrm{th}}$.

The aforementioned procedures are applied to all eRRHs in the network. Thus, the set of all possible IDNC combinations $\mathcal{A}_{e_{n}, \text { IDNC }}$ and schedules $\mathcal{S}_{e_{n}}$ in the network are $\mathcal{A}_{\text {IDNC }}=$ $\bigcup_{e_{n} \in \mathcal{K}} \mathcal{A}_{e_{n}, \text { IDNC }}$ and $\mathcal{S}=\bigcup_{e_{n} \in \mathcal{K}} \mathcal{S}_{e_{n}}$, respectively. These schedules $\mathcal{S}$ can be exactly represented by unique vertices $\mathcal{V}$ in $\mathcal{G}_{\text {IA-IDNC }}(\mathcal{V}, \mathcal{E})$ such that we transfer the subproblem $\mathrm{P} 2$ to a graph-theory based problem. Therefore, the $\mathbf{S}_{i}$-th schedule in $\mathcal{S}$ is represented by the $V_{i}$-th unique vertex in $\mathcal{G}_{\text {IA-IDNC }}(i=1,2, \cdots,|\mathcal{S}|)$. This schedule-to-vertex mapping makes any IDNC combination sent from the $e_{n}$-th eRRH with adopted rate to its corresponding associated users is decodable.

Two vertices $V_{i}$ and $V_{i \prime}$ representing two different schedules $\mathbf{S}_{i} \in \mathcal{S}_{e_{n}}$ and $\mathbf{S}_{i^{\prime}} \in \mathcal{S}_{e_{n^{\prime}}}$ are adjacent by an edge in $\mathcal{G}_{\text {IA-IDNC }}$, if the associations they represent satisfy the following condition.

- Transmission Condition (TC): $\mathrm{u} \cap \mathrm{u}^{\prime}=\phi, \forall\left(s_{1}, s_{2}\right) \in \mathbf{S}_{i} \times \mathbf{S}_{i^{\prime}}$. This condition ensures that the same user can be scheduled only to a unique eRRH.

Assuming that the power allocation of the eRRHs in the network will be computed later; then the weight of a given vertex $V$ representing a schedule $\mathbf{S}$ is expressed by

$$
w(V)=\sum_{s \in \mathbf{S}} \frac{\min _{u_{l, s} \in \mathrm{u}\left(\mathrm{f}_{e_{n}}^{c}\right)} \log _{2}\left(1+\operatorname{SINR}_{e_{n, s}, u_{l, s}}(\mathbf{P})\right)}{B} .
$$

The weight of each vertex reflects the contribution of each eRRH towards minimizing the completion time of its associated users. Actually, the transmission rate plays a crucial role in minimizing the transmission duration $T_{e_{n}}^{c}$. Thus, a larger value in (9) leads to minimize the transmission durations of delivering IDNC files to users, which minimizes their completion times. 
The design of the IA-IDNC graph makes any maximal weight clique represents a set of transmissions satisfying the following three features: i) each user is scheduled only to a single eRRH that cached one of its requested files, ii) each eRRH delivers an IDNC file with an adopted transmission rate/power that satisfies a lower completion time for a potential set of users. Such adopted rate satisfies the QoS rate guarantee and no larger than the channel capacities of all scheduled users, iii) the weight of each vertex strikes a balance between the adopted rate and the number of scheduled users to each eRRH.

The following theorem characterizes the solution of subproblem P2 based on the designed IA-IDNC graph.

Theorem 2. The transmission duration minimization subproblem P2 is equivalently represented by the maximum weight clique problem in the IA-IDNC graph, and can be expressed as

$$
=\arg \max _{\mathrm{C} \in \mathcal{C}} \sum_{V_{i} \in \mathrm{C}} w\left(V_{i}\right), \forall i=1,2, \cdots,|\mathrm{C}|,
$$

where $\mathrm{C}$ is the maximum weight clique of a maximum degree $|\mathcal{K}|$ in the IA-IDNC graph and $\mathcal{C}$ is the set of all possible maximal cliques.

Proof. The proof of Theorem 2 is omitted due to space limitation and only we provide a sketch of it as follows. First, we need to show that there is a unique one-to-one mapping between each schedule $\mathbf{S}_{i} \in \mathcal{S}$ and each vertex $V_{i} \in \mathcal{V}$ in $\mathcal{G}_{\text {IA-IDNC }}(i=1,2, \cdots,|\mathcal{S}|)$. Then, we emphasize that each maximal clique in the IA-IDNC graph that consists of a set of vertices satisfying all edge conditions represents feasible coded transmissions from the eRRHs. Finally, the proof can be concluded by showing that the contributed weight of the maximum weight clique $\mathrm{C}$ for minimizing the transmission duration is: $w(\mathrm{C})=\sum_{V_{i} \in \mathrm{C}} w\left(V_{i}\right)=\sum_{e_{n} \in \mathcal{K}} \sum_{\mathbf{S}_{i} \in \mathrm{S}} w\left(\mathbf{S}_{i}\right)=$ $\sum_{e_{n} \in \mathcal{K}} \sum_{s \in \mathbf{S}_{i}} \frac{\min _{u_{l, s} \in \mathrm{u}\left(f_{e_{n}}^{c}\right)} \log _{2}\left(1+\operatorname{SINR}_{e_{n, s}, u_{l, s}}(\mathbf{P})\right)}{B}, \forall i=1,2, \cdots,|\mathrm{C}|$, where $\mathrm{S}$ is the set of the selected potential schedules. Therefore, the subproblem P2 is equivalent to the maximum weight clique problem among the maximal cliques in the IA-IDNC graph.

The problem in Theorem 2 is clearly NP-hard problem, and solving it optimally is intractable [36]. However, we heuristically and efficiently solve it in the next subsection.

\section{B. Solution of Subproblem P2}

In this section, we solve the problem in Theorem 2 by characterizing the joint solution to the NC user scheduling and power allocation problem while designing the IA-IDNC graph. A 
proper power allocation for each eRRH leads to suppress the interference in the system, thus a better transmission rate is achieved. As a result, the transmission duration for delivering files to the scheduled users is minimized.

Consider a power-clique (PC) in $\mathcal{G}_{\text {IA-IDNC }}$ that is associated with a network-coded user scheduling $\mathbf{S}=\left\{\mathbf{S}_{1}, \mathbf{S}_{2}, \cdots, \mathbf{S}_{|K|}\right\}$, where $\mathbf{S}_{1}$ is the schedule of $e_{1}$-th eRRH, which consists of a set of associations $s_{1}, s_{2}, \cdots, s_{\left|\mathbf{S}_{1}\right|}$, and $\left|\mathbf{S}_{1}\right|=\left|\mathrm{u}\left(\mathbf{f}_{e_{n}}^{c}\right)\right|$. Our goal is to obtain a local optimal eRRH power allocation vector, denoted as $\left(P_{e_{1}}^{*}, \cdots, P_{e_{K}}^{*}\right)$ for that PC. The power allocation problem is formulated as an optimization problem of maximizing the weighed sum-rate. As such, all scheduled users receive files sent by their associated eRRHs with minimum transmission duration, which can be expressed as

$$
\begin{aligned}
& \mathrm{P} 4: \max _{P_{e_{n}}} \sum_{n=1}^{K} \frac{\left|\mathrm{u}\left(f_{e_{n}}^{c}\left(\mathbf{S}_{n}\right)\right)\right|}{B} * \min _{u_{l} \in \mathbf{u}\left(\mathfrak{f}_{e_{n}}^{c}\left(\mathbf{S}_{n}\right)\right)}\left(\log _{2}\left(1+\operatorname{SINR}_{e_{n}, u_{l}}(\mathbf{P})\right)\right), \\
& \text { s. t. } 0 \leqslant P_{e_{n}} \leqslant P_{\max }, \forall n=1,2, \cdots, K,
\end{aligned}
$$

where $\mathrm{u}\left(\mathbf{f}_{e_{n}}^{c}\left(\mathbf{S}_{n}\right)\right)$ is the set of scheduled users in $n$-th schedule corresponding to $e_{n}$-th eRRH. The power allocation problem in $\mathrm{P} 4$ is a non-convex optimization problem. Therefore, similar to the works in literature (see for example, [29], [34] and references therein), we focus on finding the local optimal solution.

The proposed solution to the problem in Theorem 2 is executed at the CBS at each transmission slot and divided into two stages: designing the IA-IDNC graph and finding its corresponding maximum PC.

First stage: The IA-IDNC graph is designed as follows. Using IDNC-C1, IDNC-C2, and $\mathbf{R C}$ conditions that explained in Section $\mathrm{V}-\mathrm{A}$, we generate all schedules $\mathcal{S}$ and represent them by vertices in $\mathcal{V}$. Afterwards, we check the connection between any two pairs $V_{i}$ and $V_{i^{\prime}}$ of vertices in $\mathcal{G}_{\text {IA-IDNC }}$ based on the transmission condition TC in Section V-A Any connected vertices result in a feasible network-coded scheduling to the eRRHs. Then, we evaluate the power allocation of such network-coded scheduling $\left\{\mathbf{S}_{1}, \mathbf{S}_{2}, \cdots, \mathbf{S}_{K}\right\}$ by solving the optimization problem (11). By the computed power allocation and corresponding rate, we compute the weights of the corresponding vertices in $\mathcal{G}_{\mathrm{IA}-\mathrm{IDNC}}$ as expressed in $(9)$. We repeat the above process to all network-coded schedules in the IA-IDNC graph.

Second stage: The second stage finds the maximum weight PC C among all other maximal PCs in $\mathcal{G}_{\text {IA-IDNC }}$ graph. In the first step, we select vertex $V_{i} \in \mathcal{V},(i=1,2, \cdots,|\mathcal{V}|)$ that has the 
maximum weight $w\left(V_{i}^{*}\right)$ and add it to $\mathrm{C}$ (at this point $\mathrm{C}=\left\{V_{i}^{*}\right\}$ ). Then, the subgraph $\mathcal{G}_{\text {IA-IDNC }}(\mathrm{C})$, which consists of vertices in graph $\mathcal{G}_{\text {IA-IDNC }}$ that are adjacent to vertex $V_{i}^{*}$ is extracted and considered for the next vertex selection process. In the next step, a new maximum weight vertex $V_{i^{\prime}}^{*}$ (i.e., $V_{i^{\prime}}^{*}$ should be in the corresponding $\mathrm{PC}$ of $\left.V_{i}^{*}\right)$ is selected from subgraph $\mathcal{G}_{\text {IA-IDNC }}(\mathrm{C})$. Now, $\mathrm{C}=\left\{V_{i}^{*}, V_{i^{\prime}}^{*}\right\}$. We repeat this process until no further vertex is adjacent to all vertices in the maximal weight $\mathrm{PC}$ C. The selected $\mathrm{C}$ contains at most $K$ vertices.

\section{New D2D Conflict Graph}

We introduce a new D2D conflict graph, denoted by $\mathcal{G}_{\mathrm{d} 2 \mathrm{~d}}(\mathcal{V}, \mathcal{E})$, that considers all possible conflicts for scheduling users on D2D links, such as transmission, network coding, half-duplex conflicts. This leads to feasible transmissions from the potential D2D transmitters $\left|\mathcal{N}_{\text {tra }}\right|$, where each $u_{k} \in \mathcal{N}_{\text {tra }}$ transmits the IDNC combination $\mathrm{f}_{u_{k}}^{d 2 d}$ to the scheduled users $\mathrm{u}\left(\mathrm{f}_{u_{k}}^{d 2 d}\right)$ with the transmission rate $r_{u_{k}}^{d 2 d}$.

Let $\mathcal{N}_{\mathrm{d} 2 \mathrm{~d}}$ denote the set of unscheduled users to the eRRHs, i.e., $\mathcal{N}_{\mathrm{d} 2 \mathrm{~d}}=\mathcal{N} \backslash \bigcup_{n=1}^{K} \mathrm{u}\left(\mathrm{f}_{e_{n}}^{c}\right)$, and let $\mathcal{N}_{\mathrm{d} 2 \mathrm{~d}, w} \subset \mathcal{N}_{\mathrm{d} 2 \mathrm{~d}}$ denote the set of users that still wants some files. Hence, the D2D conflict graph is designed by generating all vertices for $u_{k}$-th possible D2D transmitter, $\forall u_{k} \in \mathcal{N}_{\mathrm{d} 2 \mathrm{~d}}$. The vertex set $\mathcal{V}$ of the entire graph is the union of vertices of all users. Consider, for now, generating the vertices of $u_{k}$-th user. Note that $u_{k}$-th D2D transmitter can encode its IDNC file $\mathrm{f}_{u_{k}}^{d 2 d}$ using its previously received files $\mathcal{H}_{u_{k}}$. Therefore, each vertex is generated for each single file $f_{h} \in \mathcal{W}_{u_{i}} \cap \mathcal{H}_{u_{k}}$ that is requested by each user $u_{i} \in \mathcal{N}_{\mathrm{d} 2 \mathrm{~d}, w} \cap \mathcal{Z}_{u_{k}}$ and for each achievable rate $r$ of $u_{k}$-th user that is defined below.

Definition 4. The set of achievable rates $\mathcal{R}_{u_{k}, u_{i}}^{d 2 d}$ from $u_{k}$-th user to $u_{i}$-th user is a subset of achievable rates $\mathcal{R}_{u_{k}}^{d 2 d}$ that are less than or equal to channel capacity $r_{u_{k}, u_{i}}^{d 2 d}$. It can be expressed by $\mathcal{R}_{u_{k}, u_{i}}^{d 2 d}=\left\{r \in \mathcal{R}_{u_{k}}^{d 2 d} \mid r \leq r_{u_{k}, u_{i}}^{d 2 d}\right.$ and $u_{i} \in \mathcal{N}_{d 2 d, w} \cap \mathcal{Z}_{u_{k}}$ and $\left.r \geq R_{e_{n^{*}}}^{c}\right\}$.

The above definition emphasizes that $u_{i}$-th user in the coverage zone $\mathcal{Z}_{u_{k}}$ can receive a file from $u_{k}$-th D2D transmitter if the adopted transmission rate $r$ is in the achievable set $R_{u_{k}, u_{i}}^{d 2 d}$ and no less than the minimum transmission rate of $e_{n^{*}}$-th eRRH. Therefore, we generate $\left|\mathcal{R}_{u_{k}, u_{i}}^{d 2 d}\right|$ vertices for a requesting file $f_{h} \in \mathcal{H}_{u_{k}} \cap \mathcal{W}_{u_{i}}, \forall u_{i} \in \mathcal{N}_{\mathrm{d} 2 \mathrm{~d}, \mathrm{w}} \cap \mathcal{Z}_{u_{k}}$. In summery, a vertex $v_{r, i, f}^{k}$ is generated for each association of a transmitting user $u_{k}$, a rate $r \in \mathcal{R}_{u_{k}, u_{i}}^{d 2 d}$, and a requesting file 
$f_{h} \in \mathcal{H}_{u_{k}} \cap \mathcal{W}_{u_{i}}$ of user $u_{i} \in \mathcal{N}_{\mathrm{d} 2 \mathrm{~d}, \mathrm{w}} \cap \mathcal{Z}_{u_{k}}$. Similarly, we generate all vertices for all users in $\mathcal{N}_{\mathrm{d} 2 \mathrm{~d}} \cdot 1$

All possible conflict connections between vertices (conflict edges between circles) in the D2D conflict graph are provided as follows. Two vertices $v_{r, i, h}^{k}$ and $v_{r^{\prime}, i^{\prime}, h^{\prime}}^{k^{\prime}}$ are adjacent by a conflict edge in $\mathcal{G}_{\mathrm{d} 2 \mathrm{~d}}$, if one of the following conflict conditions (CC) is true:

- IDNC (CC1): $\left(u_{k}=u_{k^{\prime}}\right)$ and $\left(f_{h} \neq f_{h^{\prime}}\right)$ and $\left(f_{h}, f_{h^{\prime}}\right) \notin \mathcal{H}_{u_{k^{\prime}}} \times \mathcal{H}_{u_{k}}$. A conflict edge between vertices is connected as long as the files they represent are not-instantly decodable to a set of scheduled users to the same $u_{k}$-th D2D transmitter.

- Rate (CC2): $\left(u_{k}=u_{k^{\prime}}\right)$ and $\left(r \neq r^{\prime}\right)$. All adjacent vertices correspond to the same $u_{k}$-th D2D transmitter should have the same achievable rate.

- Transmission $(\mathbf{C C 3}):\left(u_{k} \neq u_{k^{\prime}}\right)$ and $\left(u_{i}=u_{i^{\prime}}\right)$. The same user cannot be scheduled to two different D2D transmitters $u_{k}$ and $u_{k^{\prime}}$.

- Half-Duplex (CC4): $\left(u_{k}=u_{i^{\prime}}\right)$ or $\left(u_{k^{\prime}}=u_{i}\right)$. The same user cannot transmit and receive in the same transmission slot.

Given the aforementioned designed D2D conflict graph, the following theorem reformulates the subproblem P3.

Theorem 3. The subproblem of maximizing the number of scheduled users on D2D links in $P_{3}$ at the $t$-th transmission is equivalently represented by the maximum weight independent set (IS) selection among all the maximal sets in the $\mathcal{G}_{d 2 d}$ graph, where the weight $\psi\left(v_{r, i, h}^{k}\right)$ of each vertex $v_{r, i, h}^{k}$ is given by

$$
\psi\left(v_{r, i, f}^{k}\right)=\left|\mathcal{Z}_{u_{k}} \cap \mathcal{N}_{d 2 d, w}\left(\mathcal{H}_{u_{k}}\right)\right|\left(\frac{r}{B}\right) .
$$

The above weight metric shows two potential benefits: i) $\left|\mathcal{Z}_{u_{k}} \cap \mathcal{N}_{d 2 d, w}\left(\mathcal{H}_{u_{k}}\right)\right|$ represents that the $u_{k}$-th transmitting user is connected to many other users that are requesting files in $\mathcal{H}_{u_{k}}$ and ii) $\left(\frac{r}{B}\right)$ provides a balance between the transmission rate and the number of scheduled users on D2D links.

\footnotetext{
${ }^{1}$ For the space limitation, we generate only the vertcies of $\mathcal{N}_{\mathrm{d} 2 \mathrm{~d}}$ users and ignore those representing scheduled users to $e_{n^{\prime}}$-th eRRH, $e_{n^{\prime}} \in \mathcal{K}$. However, they can be generated and connected to each other using similar steps in this section with the difference that any D2D transmitter should be able to deliver files within the idle time slot of the corresponding eRRH.
} 


\section{Details of Greedy Solution}

The proposed solution here greedily maximizes the number of scheduled users on D2D links within $T_{e_{n}}^{c}$ by maximizing the number of vertices in any IS in the D2D conflict graph. In order to maximize the number of vertices in any IS, we update the weight of each vertex. An appropriate design of the updated weights of vertices leads to selection of a large number of vertices and each vertex has high original weight that defined in (12).

Let $\pi_{v, v}{ }^{2}$ define the non-adjacency indicator of vertices $v$ and $v^{\prime}$ in the $\mathcal{G}_{\mathrm{d} 2 \mathrm{~d}}$ graph such that:

$$
\pi_{v, v^{\prime}}= \begin{cases}1 & \text { if } v \text { is not adjacent to } v^{\prime} \text { in } \mathcal{G}_{\mathrm{d} 2 \mathrm{~d}}, \\ 0 & \text { otherwise. }\end{cases}
$$

Now, let the weighted degree $n_{v}$ of vertex $v$ is defined by $n_{v}=\sum_{v^{\prime} \in \mathcal{G}_{\mathrm{d} 2 \mathrm{~d}}} \pi_{v, v^{\prime}} \cdot \psi\left(v^{\prime}\right)$, where $\psi\left(v^{\prime}\right)$ is the original weight of vertex $v^{\prime}$ defined in Proposition 3. Hence, the modified weight of vertex $v$ is defined as

$$
w(v)=\psi(v) n_{v}=\psi(v) \sum_{v^{\prime} \in \mathcal{G}_{\mathrm{d} 2 \mathrm{~d}}} \pi_{v, v^{\prime}} \cdot \psi\left(v^{\prime}\right) .
$$

The modified weight of a vertex $v$ in (14) points two attractive features: (i) it has a large original weight, and (ii) it is not adjacent to a large number of vertices that have high original weights. Based on this, we iteratively and heuristically execute a greedy vertex search scheme as follows. Initially, a vertex $v^{*}$ that has the maximum weight $w\left(v^{*}\right)$ is selected and added to the maximal IS $\Gamma$ (i.e., $\Gamma=\left\{v^{*}\right\}$ ). Then, the subgraph $\mathcal{G}_{\mathrm{d} 2 \mathrm{~d}}(\Gamma)$, which consists of vertices in graph $\mathcal{G}_{\mathrm{d} 2 \mathrm{~d}}$ that are not adjacent to vertex $v^{*}$, is extracted and considered for the next process. In the next step, a new maximum weight vertex $v^{*}$ is selected from subgraph $\mathcal{G}_{\mathrm{d} 2 \mathrm{~d}}(\Gamma)$ (at this point $\Gamma=\left\{v^{*}, v^{\prime *}\right\}$ ). We repeat this process for all transmitting users so that no further vertex is not adjacent to all the vertices in $\Gamma$. The selected D2D transmitters in the maximum IS $\Gamma$ generate coded files and broadcast them to all neighboring users on D2D links.

The overall two-solution joint approach that are explained in Section $\mathrm{V}-\mathrm{B}$ and Section $\mathrm{V}-\mathrm{D}$. respectively, is provided in Algorithm 1 .

Example: We illustrate in this example how to design the IA-IDNC and D2D conflict graphs of the network presented in Fig. 3.

\footnotetext{
${ }^{2}$ For notation simplicity, we replace $v_{r, k, l}^{i}$ by $v$ and $v_{r^{\prime}, k^{\prime}, l^{\prime}}^{i^{\prime}}$ by $v^{\prime}$.
} 


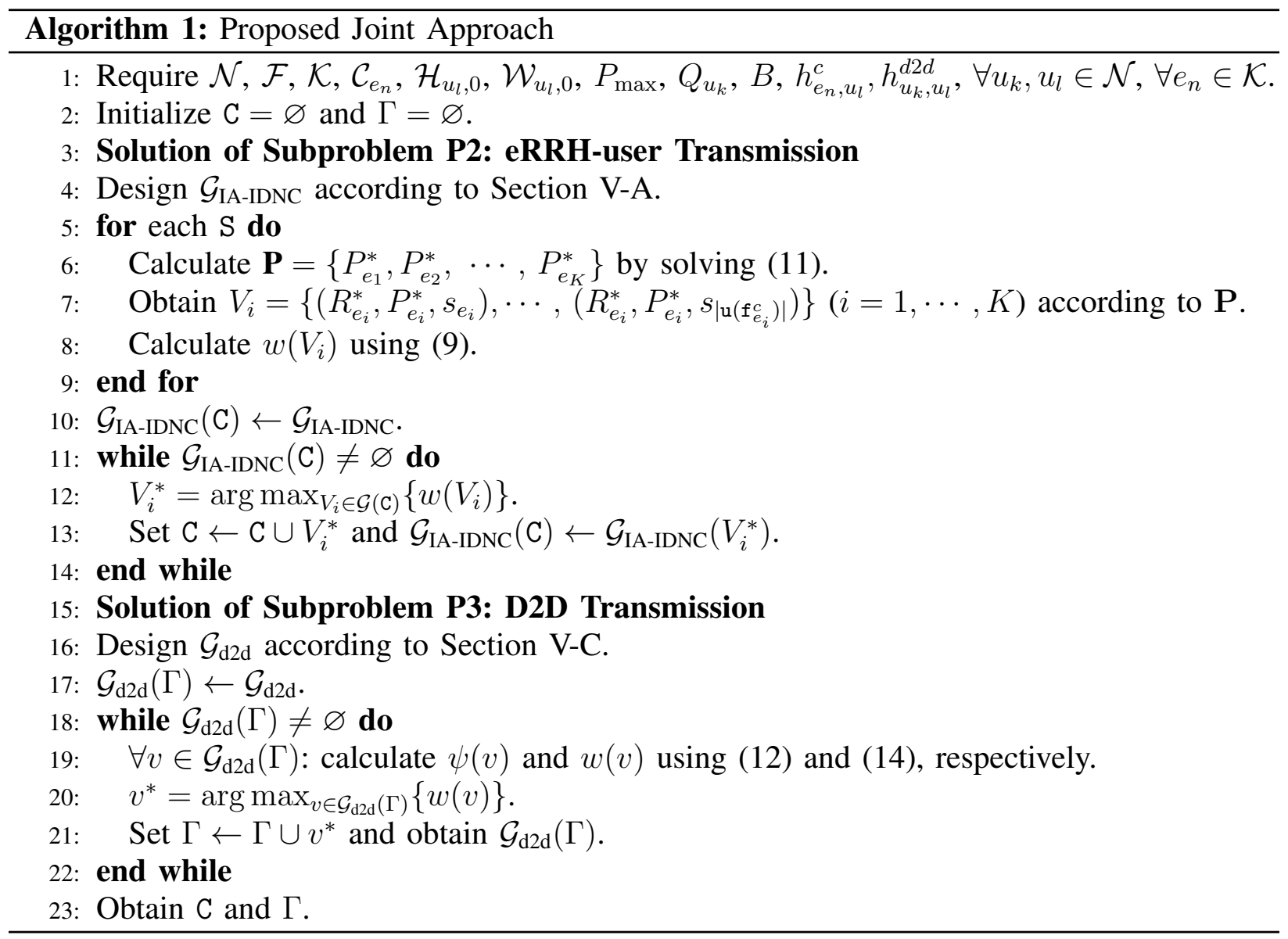

- In Fig. 4, we plot the IA-IDNC graph, where each vertex represents a possible NC combination that consists of combined associations in each eRRH. For plotting simplification, we did not include the vertices that represents one association (no NC). The connections between vertices (circles) is based on the TC condition that explained in Section $\mathrm{V}-\mathrm{A}$. There are many possible maximal PCs in $\mathcal{G}_{\text {IA-IDNC }}$ that are represented by connected vertices. Each one represents the potential network-coded scheduling of the eRRHs that minimizes the completion time of users. For example, one possible maximal PC shown in red color in Fig. 4 is $\left\{s_{R^{*} P^{*} 53}^{1}, s_{R^{*} P^{*} 61}^{1}, s_{R^{*} P^{*} 22}^{2}, s_{R^{*} P^{*} 34}^{2}\right\}$. The five indices $e_{1}, R^{*}, P^{*}, 5,3$ in the first association represent first eRRH, its transmission rate and power level, scheduled user and its requested file, respectively.

- To ease the understanding of the D2D conflict graph, we plot it only for the first three users $\left\{u_{1}, u_{2}, u_{3}\right\}$ of the network presented in Fig. 3 and irrespective to the possible scheduled users to the eRRHs. The D2D conflict graph is shown in Fig. 5, where the conflict conditions 


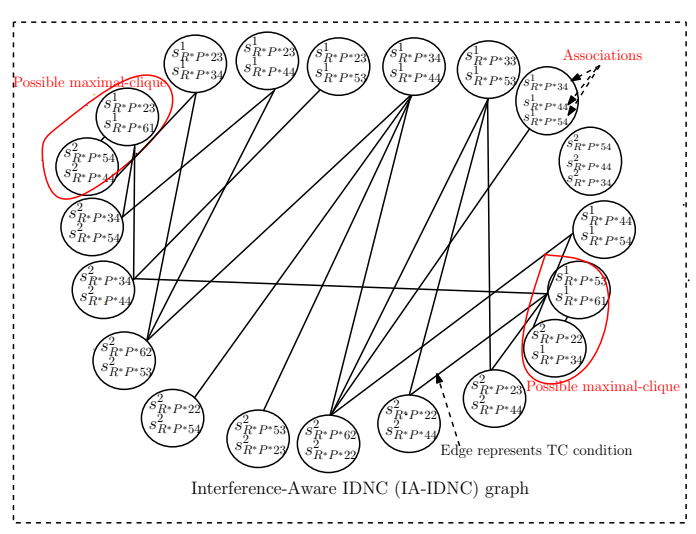

Fig. 4. The IA-IDNC graph.

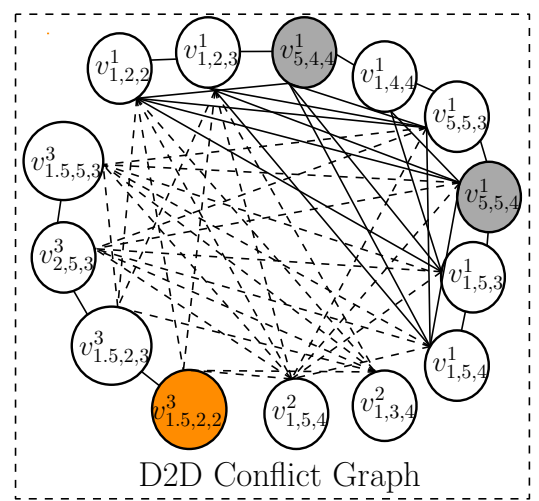

Fig. 5. The D2D conflict graph.

CC1 and $\mathbf{C C 2}$ are represented by solid lines and conditions $\mathrm{CC3}$ and $\mathrm{CC4}$ are represented by dash lines. By Theorem 3, one possible maximal IS in this graph is $\left\{v_{5,4,4}^{1}, v_{5,5,4}^{1}, v_{1.5,2,2}^{3}\right\}$. The first vertex $v_{5,4,4}^{1}$ represents the transmitting user $u_{1}$, its rate $r=5$, the scheduled user $u_{4}$ and its requested file $f_{4}$, respectively.

\section{Vi. Completion Time Minimization: Coordinated Scheduling Approach}

In this section, we propose a faster and simpler coordinated scheduling approach. The need for this approach is invoked by the possibly large number of IDNC combinations generated by the joint approach in large network size. In such large networks, we can utilize this alternative approach to obtain fast and efficient solution.

Let $P_{e_{n}}$ be a fixed power level of $e_{n}$-th eRRH, $\forall e_{n} \in \mathcal{K}$. The completion time minimization problem at $t$-th transmission slot can be written as a coordinated scheduling problem as follows

$$
\begin{aligned}
& \text { P5 : } \max _{\substack{f_{e_{n}}^{c} \subseteq \mathcal{P}\left(\mathcal{C}_{e_{n}}\right) \\
\mathrm{u}\left(\mathbf{f}_{e_{n}}^{c}\right) \cap \mathrm{u}\left(\mathbf{f}_{e_{e^{\prime}}}^{c}\right)=\varnothing \\
R_{e_{n}} \in \mathcal{R}}} Z_{t} \\
& \text { subject to }\left\{\begin{array}{l}
\left(\mathrm{u}\left(\mathrm{f}_{u_{k}}^{d 2 d}\right), r_{u_{k}}\right)=\arg \min _{\substack{\mathcal{N}_{\text {tra }} \in \mathcal{P}(\mathcal{N}) \\
\mathbf{f}_{k}^{d 2 d} \in \mathcal{P}\left(\mathcal{H}_{u_{k}}\right) \\
r_{u_{k}} \in \mathcal{R}_{u_{k}}}}\left\{\max _{u_{l} \in \mathcal{N}_{w, t}} \mathrm{~T}_{u_{l}, t}\right\}, \forall u_{k} \in \mathcal{N}_{\text {tra }, t} ; \\
\mathrm{u}\left(\mathrm{f}_{u_{k}}^{d 2 d}\right) \cap \mathrm{u}\left(\mathrm{f}_{u_{k^{\prime}}}^{d 2 d}\right)=\varnothing, \forall u_{k} \neq u_{k^{\prime}} \in \mathcal{N}_{\text {tra }, t} ; \\
\mathrm{f}_{u_{k}}^{d 2 d} \subseteq \mathcal{P}\left(\mathcal{H}_{u_{k}}\right) ; r_{u_{k}} \geq R_{\text {th }} .
\end{array}\right.
\end{aligned}
$$

The objective function $15 \mathrm{~b}$ ) of problem P5 represents the possible completion time minimization that we can obtain from D2D transmissions and (15a) represents maximizing the number of users 
left to be scheduled to eRRHs.

To solve the problem in P5, we develop a simple and fast approach that first schedules users that have low channel capacities from eRRHs on D2D links, and the remaining unscheduled users (if any) can be scheduled by eRRHs with high transmission rates. This solution not only minimizes the completion time of users, but also offloads the eRRHs' radio resources. Indeed, after D2D transmissions, few users left to be scheduled by eRRHs. This approach is summarized in Algorithm 2, which consists of the following two stages at every transmission slot: i) users experience relatively weak channels from the eRRHs should be scheduled to the potential D2D transmitters on D2D links, and ii) the eRRHs deliver encoded files to a set of users that have not previously scheduled on D2D links. The coordinated scheduling approach is described below.

First stage: Here, we focus on scheduling a set of users that have low rates from the eRRHs to a set of potential transmitters via D2D links as such we solve problem (15b) efficiently. First step: Inspired by Section $\mathrm{V}-\mathrm{C}$, we follow the same procedures that construct the new D2D conflict graph $\mathcal{G}_{\mathrm{d} 2 \mathrm{~d}}(\mathcal{V}, \mathcal{E})$. Thus, we generate a vertex $v_{r, l, f}^{k}$ for each transmitting user $u_{k}$, a transmission rate $r_{u_{k}} \in \mathcal{R}_{u_{k}, u_{i}}^{d 2 d}$ and a missing file $f_{h} \in \mathcal{H}_{u_{k}} \cap \mathcal{W}_{u_{l}}$ of a user $u_{l} \in \mathcal{N}_{w} \cap \mathcal{Z}_{u_{k}}$. Further, the rate of each transmitting user in each generated vertex should be greater than or equal to $R_{\mathrm{th}}$. Similarly, we generate the vertices for $N$ users and then connect them as in Section $\mathrm{V}-\mathrm{C}$. Second step: We design two-layer weights for each generated vertex in the $\mathcal{G}_{\mathrm{d} 2 \mathrm{~d}}$ graph, named by secondary and primary weights. The secondary weight of a vertex $v_{r, l, f}^{k}$ is defined as $w\left(v_{r, l, f}^{k}\right)=$ $\frac{r_{u_{k}}}{B}$ that shows a partial contribution of that vertex in terms of reducing the completion time in the network. The primary weigh of a vertex $v_{u_{l}, f_{h}}$ is defined as $w\left(v_{u_{i}, f_{h}}\right)=\frac{B}{\min _{e_{n} \in \mathcal{K}} R_{e_{n}, u_{l}}}, \forall f_{h} \in \mathcal{C}_{e_{n}}$. This primary weight characterizes the users based on their channel capacities from the eRRHs to give them priority to be scheduled on D2D links. A vertex with high primary weight (low rate from eRRHs) leads to prolonged file delivery time from eRRHs. Then the corresponding users of such vertices should be scheduled on D2D links with the maximum rate from any possible potential D2D transmitters. As such, the completion time of these users is minimized.

Third step: We propose to iteratively perform maximum weight search to form the set of D2D transmitters and their scheduled users in the maximal IS $\Gamma$ as follows. First, we search for the vertex with the maximum primary weight and find its corresponding maximum secondary weight. If two or more vertices have equal weights, we select one vertex randomly. We continue this process until there are no other available vertices that can be included in the selected IS. 


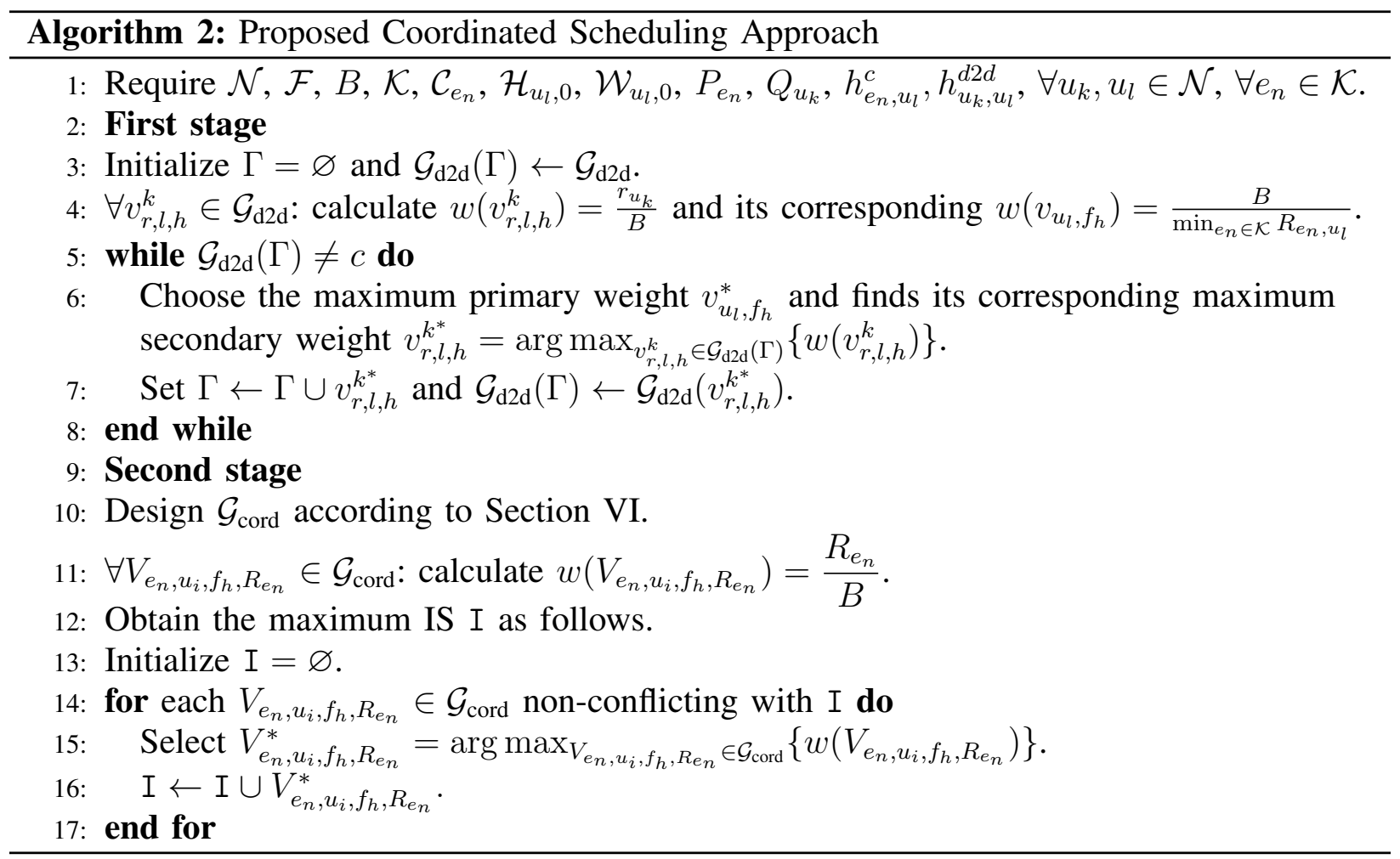

At the end, the final IS $\Gamma$ consists of vertices that represent a set of potential D2D transmitters. Each of these D2D transmitters serves users that have low channel capacities from the eRRHs.

Second stage: Here, we schedule users that are not scheduled on D2D links to eRRHs using RA-IDNC. In particular, we solve problem (15a) by maximizing the number of scheduled users to the eRRHs. First, we construct the coordinated scheduling graph, denoted by $\mathcal{G}_{\text {cord }}(\mathcal{E}, \mathcal{V})$, by generating a vertex $V_{e_{n}, u_{i}, f_{h}, R_{e_{n}}}$ for each $e_{n} \in \mathcal{K}$, for every file $f_{h}$ is requested by user $u_{i} \in$ $\mathcal{N}_{w, t} \backslash \Gamma$, and for each achievable rate for that user $R_{e_{n}} \geq r_{\min }$, where $r_{\min }$ is the minimum selected transmission rate of any transmitting user in $\Gamma$. The configuration of the set of edges in the scheduling graph is divided into coding ( $\mathrm{NC}$ and rate edges) and transmission edge. Two vertices $V_{e_{n}, u_{i}, f_{h}, R_{e_{n}}}$ and $V_{e_{n}, u_{i^{\prime}}, f_{h^{\prime}}, R_{e_{n^{\prime}}}}$ representing the same eRRH are adjacent by a conflict edge if they do not satisfy the IDNC and rate conditions in Section $\mathrm{V}-\mathrm{A}$. Similarly, two vertices $V_{e_{n}, u_{i}, f_{h}, R_{e_{n}}}$ and $V_{e_{n^{\prime}}, u_{i^{\prime}}, f_{h^{\prime}}, R_{e_{n^{\prime}}}}$ representing different eRRHs are adjacent by a conflict transmission edge if the same user $u_{i}$ is scheduled to different eRRHs, i.e., $u_{i}=u_{i^{\prime}}$ and $e_{n} \neq e_{n^{\prime}}$. Then, a maximum search process is executed in $\mathcal{G}_{\text {cord }}$ to obtain the maximum IS I as presented in Algorithm 2 . 


\section{NUMERICAL RESULTS}

This section presents selected simulation results that compare the completion time performances of our proposed two schemes with baseline algorithms. We consider a downlink D2D-

aided F-RAN system where the eRRHs have fixed locations and users are distributed randomly at every transmission within a hexagonal cell of radius $900 \mathrm{~m}$. We set the radius of the users' coverage zone $\mathrm{R}$ to $50 \mathrm{~m}$ and the number of eRRHs $K$ to 3 . We consider the SUI-Terrain type B model in which the channel model of both F-RAN and D2D communications is mostly affected by the location of the users within the cell. Path loss is calculated as $148+40 \log _{10}($ distance $[\mathrm{km}])$. We consider that the channels are perfectly estimated. The noise power and the maximum' eRRH/user power are assumed to be $-174 \mathrm{dBm} / \mathrm{Hz}$ and $P_{\max }=Q=-42.60 \mathrm{dBm} / \mathrm{Hz}$, respectively. The bandwidth is $1 \mathrm{MHz}$ and the eRRH caching ratio $\mu$ is 0.6 . As discussed in Section $\Pi$. at the beginning of the D2D-aided F-RAN transmission, each user already has about $45 \%$ and $55 \%$ of $F$ files. To assess the performances of our proposed schemes with different thresholds ( $R_{\mathrm{th} 1}=0.05, R_{\mathrm{th} 2}=0.5$, and $R_{\mathrm{th} 3}=5$ ), we simulate various scenarios with different number of users, number of files, and file sizes.

For the sake of comparison, our proposed schemes are compared with the following two baseline NC schemes.

- Random Linear NC (RLNC): In RLNC algorithm, each user is scheduled to a single eRRH to which it has the maximum channel capacity. Then, each eRRH encodes all files in its cache using random coefficients from Galois field. However, this algorithm ignores the dynamic transmission rates. To ensure successful delivery of files to users, the selected transmission rate in each $\mathrm{eRRH}$ is the minimum channel capacities of all scheduled users.

- Classical IDNC: For both eRRHs and D2D transmissions, this scheme focuses on network layer optimization, in which the coding decisions depends solely on the file combinations. For successful files' decoding, the transmission rates of both the eRRHs and transmitting users should match the minimum achievable capacity of all scheduled users.

For completeness of our work, we also compare our proposed schemes with the uncoded schemes.

- Uncoded Unicast: This scheme schedules only one user to each eRRH from which it receives an uncoded file with its maximum transmission rate. In addition, the untargeted users by the eRRHs is served by implementing uncoded D2D transmissions. 


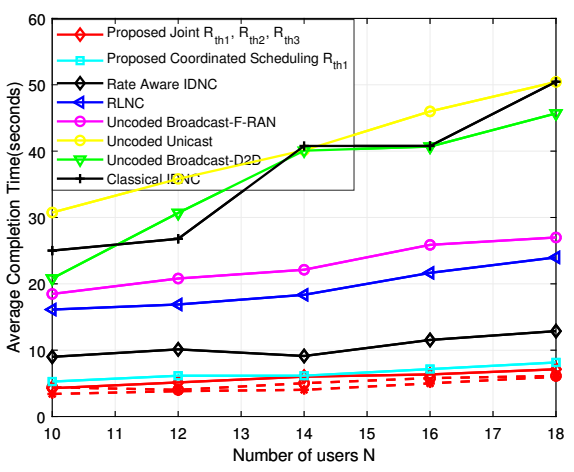

Fig. 6. Average completion time versus the number of users $N$.

- Uncoded Broadcast-F-RAN: The eRRHs broadcast uncoded files sequentially as such all users are served. In this scheme, each eRRH transmits with the lowest transmission rate of all scheduled users.

- Uncoded Broadcast-D2D: In this scheme, set of transmitting users is selected randomly to broadcast uncoded files from their Has sets that are missing at the largest number of their neighboring users. The transmission rate is selected based on the minimum transmission of all scheduled users.

Recently, RA-IDNC scheme is studied in [26] where all eRRHs use the same transmission rate that corresponds to the minimum transmission rate of all scheduled users. In addition, the unscheduled users by the eRRHs are scheduled from transmitting users over D2D links with the same rate that is used by the eRRHs. Thus, we include the RA-IDNC and compare it with the proposed schemes.

In Fig. 6, we depict the average completion time versus the number of users $N$. We consider a D2D-aided F-RAN model with a frame of 15 files and a file size of 1 Mbits. From this figure, it can be seen that the proposed schemes offer improved performance in terms of completion time reduction as compared to the other schemes. This improved performance is due to the joint and coordinated schemes that (i) judiciously schedule users, adopt the transmission rate of each eRRH and optimize the transmission power of each eRRH, and (ii) select potential users for transmitting coded files over D2D links. In particular, the uncoded unicast suffers from targeting few users that have relative strong channel qualities. As a result, a higher number of transmissions, at least $(N * F) /\left(K+\left|\mathcal{N}_{\text {tra }}\right|\right)$ transmissions, is needed for frame delivery completion, and it leads to a high completion time. Uncoded broadcast schemes suffer from 
serving all users at the cost of adopting the transmission rate of all eRRHs and transmitting users with the minimum transmission rate of the served users. Furthermore, uncoded broadcast D2D scheme offers a poor completion time performance as all transmitting users do not benefit from the transmission, i.e., they cannot transmit and receive at the same time. RLNC is a rate-less scheme that targets all users by sending encoded file with the lowest rate of all users. On the other hand, RA-IDNC scheme offers an improved performance compared to uncoded, RLNC, and classical IDNC schemes as mentioned in [26]. This is because the coding decisions in RAIDNC scheme not only depends on the file combinations, but also on the channel qualities of the scheduled users. This effectively balances between the number of scheduled users and the transmission rate of eRRHs/transmitting users. However, selecting one transmission rate (the minimum rate) for all eRRHs and transmitting users degrades the completion time performance of the RA-IDNC scheme. This is a clear limitation of the RA-IDNC scheme in [23], as it does not fully exploit the typical variable channel qualities of the different eRRHs/transmitting users to their scheduled users. Our proposed joint and coordinated schemes fully utilize the eRRHs and transmitting users' resources to choose their own transmission rates, XOR combinations, and scheduled users. Consequently, a better performance of our proposed schemes compared to the RA-IDNC scheme is achieved. Moreover, the joint scheme optimizes the employed rates using power control on each eRRH. Thus, it works better than our proposed coordinated scheme. Note that the completion time performances of the classical IDNC and uncoded broadcast D2D schemes are of orders $10^{5}$ and $10^{3}$, respectively. Thus, we omit them from all the remaining figures.

In Fig. 7, we show the average completion time versus the number of files $F$. The simulated D2D-aided F-RAN system composed of 15 users and file size of 1 Mbits. Again, for the abovementioned reasons in Fig. 6, our proposed schemes outperform the other schemes. It can be observed from the figure that increasing the frame size leads to an increasing in the completion time of all schemes. The opportunities of mixing files using NC in the RA-IDNC and proposed schemes are limited with few files. Therefore, all NC schemes have roughly similar performances. As the number of files increases, the increase in the completion time with our proposed schemes is low. This is in accordance with our results in Theorem 2 and Theorem 3, where it is shown that our proposed schemes judiciously allow each eRRH and each transmitting user to decide on a set of files to be XORed. As such, they are beneficial to a significant set of users that have 

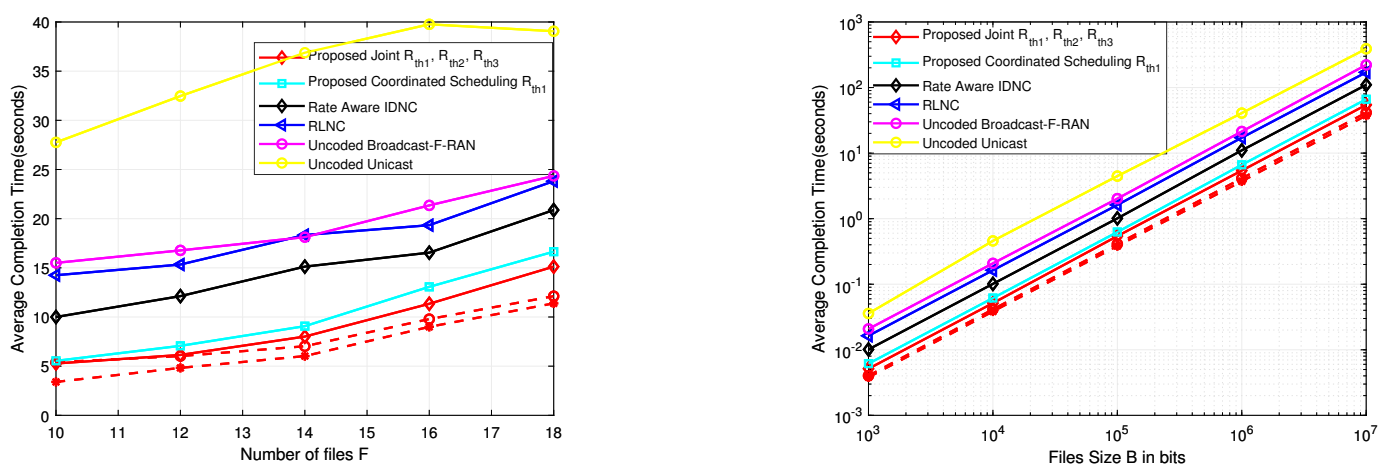

Fig. 7. Average completion time versus the number of files $F$. Fig. 8. Average completion time versus file size $B$.

relatively good channel qualities. Even though uncoded broadcast and RLNC schemes completes file transmissions in fewer transmissions ( $F$ transmissions) than our proposed schemes, each of their transmission durations is longer than a single transmission of the proposed schemes. Thanks to their optimized higher rate/power and users' transmission that result in less completion time.

In Fig. 8, we illustrate the impact of increasing the file size $B$ on the average completion time. In this figure, we simulate the D2D-aided F-RAN system composed of 12 users and 15 files. We can observe that the performances of all schemes increase linearly with the file size. This is in accordance with the completion time expression in Corollary 1, where it was emphasized that $\mathrm{T}_{o}$ increases linearly with $B$. From physical-layer view, as $B$ increases, more bits are needed for delivering files. Thus, time delay is increased to receive files from eRRHs/transmitting users.

Finally, some insights from our presented numerical results are given as follows. First, it is advantageous to serve many users with $\mathrm{NC}$ files as in the classical IDNC algorithm, but selecting the minimum transmission rate of all scheduled users degrades its performance. Thus, this scheme is impractical from physical-layer perspective. Second, although the uncoded unicast scheme uses the maximum transmission rate of each user, it needs a large number of transmissions for completion. Thus, its completion time performance is degraded. Third, RA-IDNC scheme overcomes the limitations of the aforementioned schemes but suffers from selecting the lowestrate of the fixed-power eRRHs in the system. This limitation further degrades the completion time performance of the RA-IDNC scheme in large network sizes with large number of eRRHs. This due to the fact that RA-IDNC always selects the lowest-rate of all eRRHs. Conversely, our transmission framework is more practically relevant as it enables different transmission rates from different eRRHs/transmitting users and optimizes the employed rates using power control 
on each eRRH.

\section{CONCLUSION}

We have developed a framework that exploits the cached contents at eRRHs, their transmission rates/powers, and previously received contents by different users to deliver the requesting contents to users with a minimum completion time in D2D-aided F-RAN. Towards this target, we have first formulated an optimization problem that seeks to minimize the completion time of users and decomposed it into two subproblems. Specifically, the first subproblem is to minimize the transmission durations of eRRHs. To solve it, we have designed an interference-aware IDNC graph that considers network-coded scheduling and power allocation for each eRRH. Based on this solution, the second subproblem maximizes the number of unscheduled users to eRRHs via D2D links. Then, we have introduced a new D2D conflict graph that achieves an effective solution to the second subproblem based on greedy search method. The aforementioned graphbased solutions of the corresponding subproblems are referred to a joint approach. For the high implementation complexity of the joint approach in large networks, we have developed an alternative and efficient coordinated scheme that has relatively low implementation complexity. Simulation results have shown that our proposed schemes can effectively minimize the frame delivery time as compared to conventional schemes.

\section{REFERENCES}

[1] A. Checko et al., "Cloud RAN for mobile networks-A technology overview," IEEE Commun. Surveys Tuts., vol. 17, no. 1, pp. 405-426, 1st Quart., 2015.

[2] M. Peng, Y. Li, Z. Zhao, and C. Wang, "System architecture and key technologies for 5G heterogeneous cloud radio access networks," IEEE Netw., vol. 29, no. 2, pp. 6-14, Mar. 2015.

[3] Z. Yang, Z. Ding, and P. Fan, "Performance analysis of cloud radio access networks with uniformly distributed base stations," IEEE Trans. Veh. Technol., vol. 65, no. 1, pp. 472-477, Jan. 2016.

[4] Y. Cai, F. R. Yu, and S. Bu, "Cloud computing meets mobile wireless communications in next generation cellular networks," IEEE Netw., vol. 28, no. 6, pp. 54-59, Nov. 2014.

[5] S.-H. Park, O. Simeone, O. Sahin, and S. Shamai, "Joint precoding and multivariate backhaul compression for the downlink of cloud radio access networks,” IEEE Trans. Signal Process., vol. 61, no. 22, pp. 5646-5658, Nov. 2013.

[6] A. Douik, H. Dahrouj, T.-Y. Al-Naffouri, and M.-S. Alouini, "Coordinated scheduling and power control in cloud-radio access networks," IEEE Trans. on Wireless Commun., vol. 15, no. 4, pp. 2523-2536, Apr. 2016.

[7] M. Peng, C. Wang, V. Lau, and H. V. Poor, "Fronthaul-constrained cloud radio access networks: Insights and challenges," IEEE Wireless Commun., vol. 22, no. 2, pp. 152-160, Apr. 2015. 
[8] R. Tandon and O. Simeone, "Harnessing cloud and edge synergies: Toward an information theory of fog radio access networks," IEEE Commun. Mag., vol. 54, no. 8, pp. 44-50, Aug. 2016.

[9] A. Asadi, Q. Wang, and V. Mancuso, “A survey on device-to-device communication in cellular networks," IEEE Commun. Surveys Tuts., vol. 16, no. 4, pp. 1801-1819, 2014.

[10] Roy Karasik, O. Simeone, and S. Shamai, "How much can D2D communication reduce content delivery latency in fog networks with edge caching?," IEEE Trans. on Commun., Early Access, Dec. 2019.

[11] R. Ahlswede, N. Cai, S.-Y. Li, and R. Yeung, "Network information flow," IEEE Transactions on Information Theory, vol. 46, no. 4, pp. 1204-1216, Jul. 2000.

[12] S. Sorour and S. Valaee, "Completion delay minimization for instantly decodable network codes," IEEE/ACM Trans. Netw., vol. 23 , no. 5, pp. 1553-1567, Oct. 2015.

[13] A. Douik, M.-S. Al-Abiad, and Md. J. Hossain, "An improved weight design for unwanted packets in multicast instantly decodable network Coding," IEEE Commun. Lett., vol. 23, no. 11, pp. 2122-2125, Nov. 2019.

[14] M. S. Karim, P. Sadeghi, S. Sorour, and N. Aboutorab, "Instantly decodable network coding for real-time scalable video broadcast over wireless networks," EURASIP J. Adv. Signal Process., vol. 2016, no. 1, p. 1, Jan. 2016.

[15] T. A. Courtade and R. D. Wesel, "Coded cooperative data exchange in multihop networks," IEEE Trans. Inf. Theory, vol. 60, no. 2, pp. 1136-1158, Feb. 2014.

[16] N. Aboutorab, and P. Sadeghi "Instantly decodable network coding for completion time or delay reduction in cooperative data exchange systems," IEEE Trans. on Vehicular Tech., vol. 65, no. 3, pp. 1212-1228, Mar. 2016.

[17] S. E. Tajbakhsh and P. Sadeghi, "Coded cooperative data exchange for multiple unicasts," in Proc. of 2012 IEEE Inf. Theory Workshop, Lausanne, 2012, pp. 587-591.

[18] A. Douik, and S. Sorour, "Data dissemination using instantly decodable binary codes in fog radio access networks," IEEE Trans. on Commun., vol. 66, no. 5, pp. 2052-2064, May 2018.

[19] A. Douik, S. Sorour, T. Y. Al-Naffouri, and M.-S. Alouini, "Instantly decodable network coding: From centralized to device-to-device communications," IEEE Commun. Surveys Tuts., vol. 19, no. 2, pp. 1201-1224, 2nd Quart., 2017.

[20] A. Douik, S. Sorour, T.-Y. Al-Naffouri, and M.-S. Alouini, "Rate aware instantly decodable network codes," IEEE Trans. on Wireless Commun., vol. 16, no. 2, pp. 998-1011, Feb. 2017.

[21] X. Wang, C. Yuen, and Y. Xu, "Coding based data broadcasting for time critical applications with rate adaptation", IEEE Trans. on Vehicular Tech., vol. 63, no. 5, pp. 2429-2442, Jun. 2014.

[22] M. S. Karim, A. Douik, S. Sorour and P. Sadeghi, "Rate-aware network codes for completion time reduction in deviceto-device communications," in Proc. of 2016 IEEE Intern. Conf. on Commu. (ICC), Kuala Lumpur, 2016, pp. 1-7.

[23] M. S. Al-Abiad, A. Douik, S. Sorour, and Md.-J. Hossain, "Throughput maximization in cloud radio access networks using network coding," in Proc. of 2018 IEEE Intern. Conf. on Commun. Works. (ICCWorkshops), Kansas, MO, 2018, pp. 1-6.

[24] M.-S. Al-Abiad, S. Sorour, and Md. J. Hossain, "Cloud offloading with QoS provisioning using cross-layer network coding," IEEE Globecom'18, Abu Dhabi, UAE, 2018, pp. 1-6.

[25] M.-Saif, A. Douik, and S. Sorour, "Rate aware network codes for coordinated multi base-station networks," 2016 IEEE International Conference on Commun. (ICC) Kuala Lumpur, 2016, pp. 1-7.

[26] M.-S. Al-Abiad, A. Douik, and S. Sorour, "Rate aware network codes for cloud radio access networks," IEEE Trans. on Mobile Comp., vol. 18, no 8, pp 1898-1910, Aug. 2019.

[27] M.-S. Karim, A. Douik, and S. Sorour, "Rate-aware network codes for video distortion reduction in point-to-multipoint networks, "IEEE Trans. on Vehicular Tech., vol. 66, no. 8, pp. 7446-7460, Aug. 2017. 
[28] M. S. Al-Abiad, M. J. Hossain, and S. Sorour, "Cross-layer cloud offloading with quality of service guarantees in FogRANs," in IEEE Trans. on Commun., vol. 67, no. 12, pp. 8435-8449, Jun. 2019.

[29] H. Dahrouj, W. Yu, and T. Tang, "Power spectrum optimization for interference mitigation via iterative function evaluation," EURASIP J. Wireless Commun. Netw., vol. 2012, no. 1, pp. 1-14, 2012.

[30] J. Huang, V.-G. Subramanian, R. Agrawal, and R. Berry, "Downlink scheduling and resource allocation for OFDM systems," in Process of Conference Info. Science Sys. (CISS), March 2006.

[31] J. Huang, V.-G. Subramanian, R. Agrawal, and R. Berry, "Joint scheduling and resource allocation in uplink OFDM systems for broadband wireless access networks," in IEEE J. Sel. Top. Signal Proc., vol. 27, no. 2, pp. 226-234, Feb. 2009.

[32] A. Le, A.-S. Tehrani, A.-G. Dimakis, and A. Markopoulou, "Instantly decodable network codes for real-time applications", in Proc. of 2013 International Symposium on Network Coding (NetCod), Calgary, AB, Canada, pp 1-6, Jun. 2013.

[33] W. Yu, T. Kwon, and C. Shin, "Joint scheduling and dynamic power spectrum optimization for wireless multicell networks," in Proc. of 2010 44th Annual Conf. on Inf. Sciences and Systems (CISS' 2010), Princeton, USA,, pp. 1-6, Mar. 2010.

[34] S. S. Christensen, R. Agarwal, E. De Carvalho, and J. M. Cioffi, "Weighted sum-rate maximization using weighted MMSE for MIMO-BC beamforming design,” IEEE Trans. Wireless Commun., vol. 7, no. 12, pp. 4792-4799, 2008.

[35] D. B. West et al., Introduction to graph theory. Prentice hall Upper Saddle River, 2001, vol. 2.

[36] M. G. and D. J., “Computers and Intractability - A Guide to the theory of NP-completeness," Freeman, New York, 1979.

[37] K. Ya. and S. Masuda, "A new exact algorithm for the maximum weight clique problem," in Proc. Of the 23rd Intern. Technical Conf. on Circuits/Systems, Computers and Commun. (ITCCSCC'08), Yamaguchi, Japan.

[38] P. R. J. Ostergard, “A fast algorithm for the maximum clique problem,” Discrete Appl. Math, vol. 120, pp. 197-207. 